ARTICLE

https://doi.org/10.1038/s41467-019-14199-7

\title{
Self-assembled single-atom nanozyme for enhanced photodynamic therapy treatment of tumor
}

Dongdong Wang (10 1,5, Huihui Wu²,5, Soo Zeng Fiona Phua1, Guangbao Yang1, Wei Qi Lim¹, Long Gu1, Cheng Qian1, Haibao Wang ${ }^{3}$, Zhen Guo ${ }^{2 \star}$, Hongzhong Chen ${ }^{1} \&$ Yanli Zhao (1) ${ }^{1,4 \star}$

Hypoxia of solid tumor compromises the therapeutic outcome of photodynamic therapy (PDT) that relies on localized $\mathrm{O}_{2}$ molecules to produce highly cytotoxic singlet oxygen $\left({ }^{1} \mathrm{O}_{2}\right)$ species. Herein, we present a safe and versatile self-assembled PDT nanoagent, i.e., OxgeMCC-r single-atom enzyme (SAE), consisting of single-atom ruthenium as the active catalytic site anchored in a metal-organic framework $\mathrm{Mn}_{3}\left[\mathrm{Co}(\mathrm{CN})_{6}\right]_{2}$ with encapsulated chlorin e6 (Ce6), which serves as a catalase-like nanozyme for oxygen generation. Coordination-driven self-assembly of organic linkers and metal ions in the presence of a biocompatible polymer generates a nanoscale network that adaptively encapsulates $\mathrm{Ce} 6$. The resulted OxgeMCC-r SAE possesses well-defined morphology, uniform size distribution and high loading capacity. When conducting the in situ $\mathrm{O}_{2}$ generation through the reaction between endogenous $\mathrm{H}_{2} \mathrm{O}_{2}$ and single-atom Ru species of OxgeMCC-r SAE, the hypoxia in tumor microenvironment is relieved. Our study demonstrates a promising self-assembled nanozyme with highly efficient single-atom catalytic sites for cancer treatment.

\footnotetext{
${ }^{1}$ Division of Chemistry and Biological Chemistry, School of Physical and Mathematical Sciences, Nanyang Technological University, 21 Nanyang Link, Singapore 637371, Singapore. ${ }^{2}$ Anhui Key Laboratory for Cellular Dynamics and Chemical Biology, School of Life Sciences, University of Science and Technology of China, Hefei 230027, P. R. China. ${ }^{3}$ Radiology Department of the First Affiliated Hospital of Anhui Medical University, Hefei 230022, P. R. China. ${ }^{4}$ School of Materials Science and Engineering, Nanyang Technological University, Singapore 639798, Singapore. ${ }^{5}$ These authors contributed equally: Dongdong Wang, Huihui Wu. *email: zhenguo@ustc.edu.cn; zhaoyanli@ntu.edu.sg
} 
$\mathrm{P}$ hotodynamic therapy (PDT) has been selected as a clinical method for treating a wide range of superficial and localized cancer and other diseases since it utilizes excitation light, molecular oxygen $\left(\mathrm{O}_{2}\right)$, and photosensitizer to generate high cytotoxic singlet oxygen $\left({ }^{1} \mathrm{O}_{2}\right)$ species ${ }^{1-4}$. Possessing temporal and spatial management over localization of the light irradiation, PDT could significantly enhance therapeutic efficiency and remarkably reduce side effect, thus showing superior advantages compared to conventional radiotherapy, chemotherapy, and surgery modalities ${ }^{5-7}$. However, PDT efficiencies are seriously compromised by solid tumor hypoxia due to the uncontrollable tumor growth and also the dysregulated formation of tumor blood vessels ${ }^{8,9}$. In addition, PDT also induce microvascular collapse which would block $\mathrm{O}_{2}$ transport and further aggravate the hypoxia tumor microenvironment. Consequently, this situation leads to a vicious cycle, as PDT induces tumor hypoxia, and tumor hypoxia in turn compromises PDT outcomes ${ }^{10-12}$. To solve this problem, one of the popular approaches is to design smart nanoplatforms for localized generation of $\mathrm{O}_{2}$ within tumor sites based on highly expressed intracellular $\mathrm{H}_{2} \mathrm{O}_{2}\left(5 \mathrm{nmol} / 10^{5}\right.$ cells $\left.\mathrm{h}^{-1}\right)^{13-15}$. Various kinds of nanoplatforms such as $\mathrm{MnO}_{2}$, $\mathrm{CaO}_{2}$, carbon dots and biological catalase have been constructed to catalyze $\mathrm{H}_{2} \mathrm{O}_{2}$ into $\mathrm{O}_{2}$ for ameliorating tumor hypoxia ${ }^{16-21}$. While these nanoplatforms have shown the performance on alleviating the tumor hypoxia condition to some degree, low photosensitizer loading capacity (usually below $5 \mathrm{wt} \%$ ) resulted in high dose usage of nanoplatforms has precluded their further clinical applications. Furthermore, rapid $\mathrm{pH}$-responsive degradation of $\mathrm{MnO}_{2}$ that was thought to be advantageous because of its application for magnetic resonance (MR) imaging may be a shortcoming, since this translates to short-lived $\mathrm{O}_{2}$-generation ${ }^{22}$. Thus, developing nanoplatforms to provide a continuous $\mathrm{O}_{2}$ supply for long-term hypoxia amelioration is still a challenging task yet to be solved. There is a critical need for construction of more biocompatible PDT platforms with the ability to catalyze $\mathrm{O}_{2}$ generation continuously without self-consumption.

Recently, single-atom nanocatalysts with isolated active metal centers anchored on solid supports presented new breakthroughs in cost-effective catalysis ${ }^{23,24}$. The well-defined atom level dispersion could promote the atom economy for the metal usage through maximizing the atom utilization efficiency. Moreover, unique structure and coordination environment endow these materials with enhanced catalytic activity in many reactions with superior stability ${ }^{25-27}$. Therefore, the construction of single-atom catalysts is a powerful strategy for organismic biochemical reactions due to the highest atom utilization and abundant active sites offered. For instance, Liang and coworkers reported single-atom gold anchored carbon dots as a mitochondrial reactive oxygen species (ROS) amplifier for enhanced cancer treatment ${ }^{28}$. Xu et al. explored a single-atom zinc-based nanozyme for wound antibacterial applications ${ }^{29}$. Thus, developing optimal supporting materials with high catalyst loading, good biocompatibility, and low toxicity has still been sought after by scientists. In preference to conventional catalyst supports, nanoscale metal-organic frameworks (MOFs), constituted by metal nodes/ions and organic bridging linkers, have emerged as promising supports on account of their well-defined coordination network and tunable pore size $^{30-34}$. The possibility of constructing single-atom nanocatalysts using MOFs as supporting materials was explored. Farha and coworkers reported MOF-supported single-metal-atom vanadium species with superior catalytic activities toward 4methoxybenzyl alcohol ${ }^{35}$. As a subclass of MOFs, Prussian blue analogues (PBAs) with distinctive cubic $\mathrm{M}_{\mathrm{a}}\left[\mathrm{M}_{\mathrm{b}}(\mathrm{CN})_{6}\right]$ skeleton have been thoroughly studied because of their facile and mild synthesis as well as intrinsic multifunctional properties. $\mathrm{Mn}_{3}[\mathrm{Co}$ $\left.(\mathrm{CN})_{6}\right]_{2}(\mathrm{MC})$, a type of PBAs, with an established double- perovskite framework where $\mathrm{Mn}$ is coordinated to six neighboring nitrogen exhibits excellent $\mathrm{MR}$ imaging ability due to highspin $\mathrm{Mn}-\mathrm{N}_{6}(\mathrm{~S}=5 / 2)$ octahedra structure in the skeleton ${ }^{36}$. Many studies demonstrated that noble metal elements such as $\mathrm{Pt}$, $\mathrm{Pd}$ and Ir could partially substitute the $\mathrm{M}_{\mathrm{a}}$ or $\mathrm{M}_{\mathrm{b}}$ position without perturbing the $\mathrm{M}_{\mathrm{a}}\left[\mathrm{M}_{\mathrm{b}}(\mathrm{CN})_{6}\right]$ framework for the construction of high performance electrochemical catalysts ${ }^{37-39}$. It was also reported that noble metal nanoparticles such as $\mathrm{Ru}, \mathrm{Pt}$ and $\mathrm{Au}$ nanoparticles have catalase-like nanozyme activities ${ }^{40-42}$. In addition, the polarizable $\pi$-electron cloud of $\left[\mathrm{Co}(\mathrm{CN})_{6}\right]$ may have a certain affinity to cargos with conjugated structures ${ }^{43}$. Thus, during organic linker-metal coordination process, various bioactive molecules could be conveniently encapsulated, indicating that the metal-organic coordination might be an efficient approach for incorporating single-atom catalytic active sites and therapeutic agents in the assembled materials.

In this work, by using $\mathrm{Mn}_{3}\left[\mathrm{Co}(\mathrm{CN})_{6}\right]_{2} \mathrm{MOF}$ as the support material, we incorporate single-atom $\mathrm{Ru}$ into the framework with the loading weight ratio of up to $2.23 \mathrm{wt} \%$, where $\mathrm{Ru}$ partially substitutes Co to serve as single-atom catalytic site for endogenous oxygen generation. Facilitated by collective coordination and other noncovalent interactions (Fig. 1), organic ligand, metal ions, and chlorin e6 (Ce6) photosensitizer encapsulated by biocompatible poly-vinylpyrrolidone (PVP) polymer could selfassemble to produce the well-defined and uniform single-atom enzyme (OxgeMCC-r SAE). Some of the advantages of OxgenMCC-r SAE include high Ce6 loading capacity due to intrinsic porous property of the MOF, high catalytic ability, and high catalytic durability for rapid $\mathrm{O}_{2}$ generation from endogenous $\mathrm{H}_{2} \mathrm{O}_{2}$ without being self-consumed or requiring external activation. The high catalytic activity of this nanozyme should be attributed to six unsaturated $\mathrm{Ru}-\mathrm{C}_{6}$ coordination sites, leading to rapid decomposition of $\mathrm{H}_{2} \mathrm{O}_{2}$ with atomic economy to overcome the tumor hypoxia. $\mathrm{T}_{1}$-weighted $\mathrm{MR}$ imaging is also achieved using the resultant OxgeMCC-r SAEs on account of the existence of high-spin $\mathrm{Mn}-\mathrm{N}_{6}(\mathrm{~S}=5 / 2)$ species, which permits in vivo tracking of the therapeutic agent.

\section{Results}

Preparation and characterization. As illustrated in Fig. 1, the process for in situ one-pot multicomponent self-assembly of OxgeMCC-r SAEs involves collective coordination, hydrophobic, and electrostatic interactions among organic linker, PVP polymer, photosensitizer, and metal ions. A milk-white colloidal suspension generated immediately upon adding solution of organic $\left[\mathrm{Co}(\mathrm{C} \equiv \mathrm{N})_{6}\right]$ bridging ligand $\left(1.54 \mathrm{mg} \mathrm{mL}^{-1}\right)$ into solution of $\mathrm{Mn}^{2+}\left(0.59 \mathrm{mg} \mathrm{mL}^{-1}\right)$ in the presence of surfactant PVP, indicating the formation of $\mathrm{Mn}_{3}\left[\mathrm{Co}(\mathrm{CN})_{6}\right]_{2}$ nanoparticles (designated as MC) (Fig. 2a) ${ }^{44}$. Scanning electron microscopy (SEM) and transmission electron microscopy (TEM) results showed nearly spherical MC nanoparticles with an average size distribution of $\sim 80 \mathrm{~nm}$ (Fig. $2 \mathrm{~b}, \mathrm{c}$ ). Upon the addition of $\mathrm{Ru}^{3+}$ $\left(0.072 \mathrm{mg} \mathrm{mL}^{-1}\right)$ to the initial solution, partial substitution of Co was achieved due to stronger coordination ability between the terminal carbon of the ligand and $\mathrm{Ru}$ as compared with $\mathrm{Co}^{39}$. Essentially, $\mathrm{Ru}$ was doped in the framework via a post-exchange reaction ${ }^{37}$. Then, the doped $\mathrm{Ru}$ nodes were reduced under a mild reduction environment with low concentration $\mathrm{NaBH}_{4}$ as a reducing agent. The obtained $\mathrm{Mn}_{3}\left[\mathrm{Co}(\mathrm{CN})_{6}\right]_{2}-\mathrm{Ru}$ (MC-r) nanoparticles also showed an uniform size of about $80 \mathrm{~nm}$ with darker TEM contrast on account of the existence of high- $\mathrm{Z} \mathrm{Ru}$ atom. Herein, Ce6 was chosen as a hydrophobic photosensitizer, which could be incorporated within the MC or MC-r nanoparticles during the multicomponent self-assembly process. Adding an aqueous solution of organic $\left[\mathrm{Co}(\mathrm{C} \equiv \mathrm{N})_{6}\right]$ linker into a mixed 
a

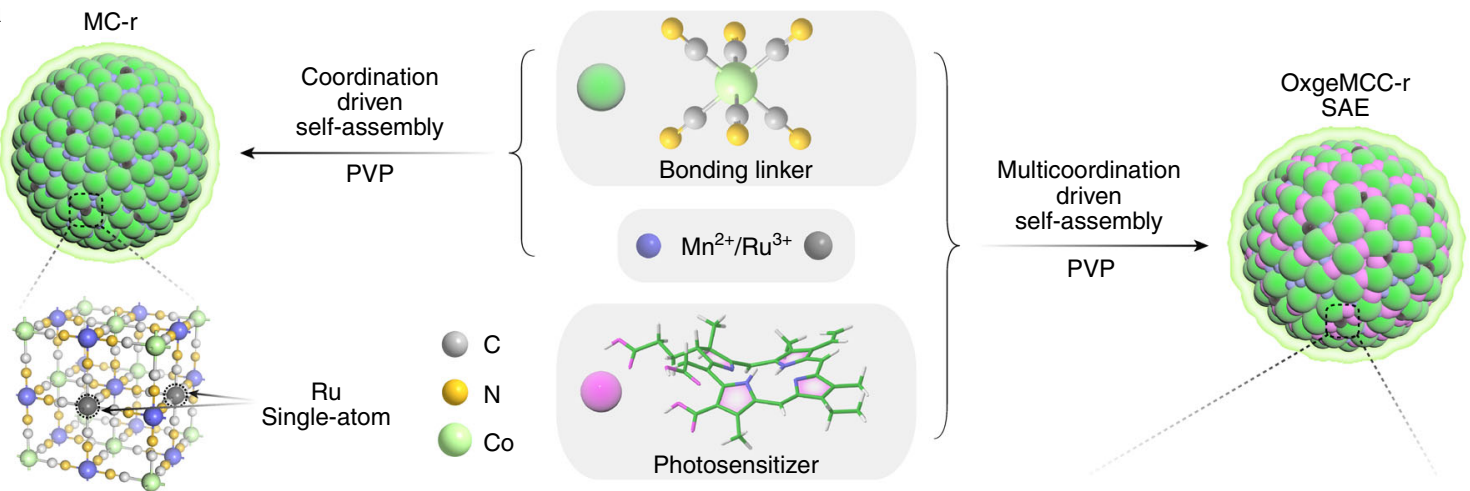

b

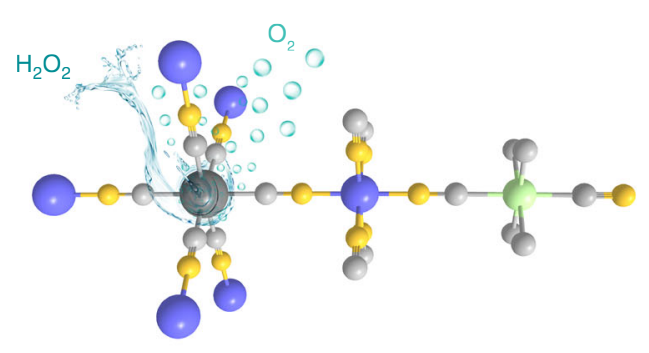

d

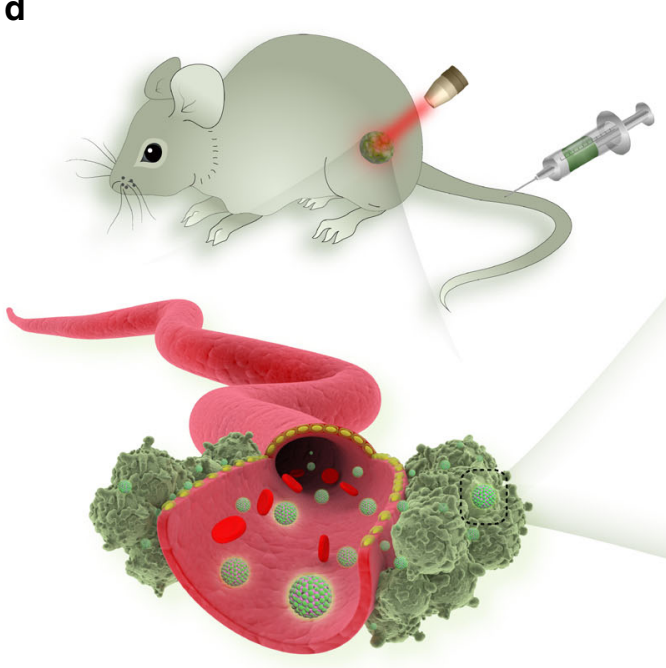

C
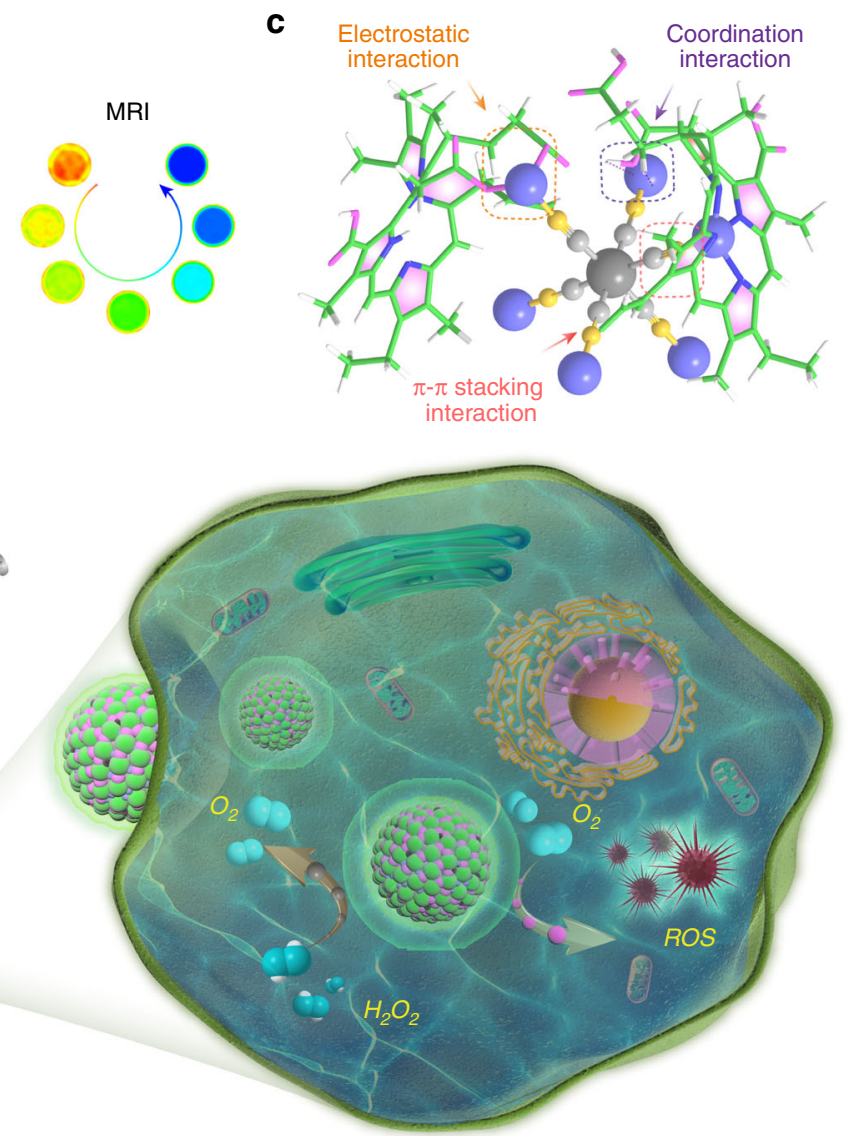

Fig. 1 Design of OxgeMCC-r nanozyme with single-atom Ru for cancer treatment. a Schematic illustration of OxgeMCC-r. OxgeMCC-r consists of catalytically active single-atom Ru site anchored in MCC with outer PVP protection layer. b Partial molecular structure of OxgeMCC-r with active singleatom Ru site serving as catalase-like nanozyme for oxygen generation. c Multicomponent coordination interactions within the OxgeMCC-r SAE. d Scheme of continuously catalytic oxygen generation and ROS production for enhanced PDT of cancer by OxgeMCC-r SAE.

solution of Ce6 $\left(0.86 \mathrm{mg} \mathrm{mL}^{-1}\right)$ and $\mathrm{Mn}^{2+}$ yielded dark-green MC-Ce6 (named as MCC), while the addition of Ce6 into a mixed aqueous solution of $\mathrm{Mn}^{2+}$ and $\mathrm{Ru}^{3+}$ afforded black-green OxgeMCC-r SAEs. SEM and TEM images also revealed that MCC and OxgeMCC-r were crack-free nanoparticles with the diameter of about $80 \mathrm{~nm}$.

The color of the MCC, MC-r, and OxgeMCC-r powder changing from light-green to dark-green and then to black-green also confirmed the stepwise incorporation of $\mathrm{Ce} 6$ and the $\mathrm{Ru}$ substitution (Fig. 3a). Powder X-ray diffraction (PXRD) patterns showed that the obtained MC, MCC, MC-r, and OxgeMCC-r were of high crystallinity with sharp diffraction peaks (Fig. 3b). The appearance of major XRD peaks suggests that MC maintains its original framework structure during the $\mathrm{Ru}$ doping process, which is consistent with the unchanged morphology after the substitution reaction. Moreover, a slight shift to lower angle of the peak position centered at $2 \theta \sim 17^{\circ}$ was observed for MC-r and OxgeMCC-r as compared with that of MC and MCC, respectively (Fig. 3c). This phenomenon also indicates that some Co sites are substituted by bigger $\mathrm{Ru}$ during the self-assembly process. In addition, no additional diffraction peak suggests the absence of crystal Ce6 or Ru-based species in the OxgeMCC-r SAEs. These isolated $\mathrm{Ru}$ ions in the framework could serve as active catalytic sites for the decomposition of endogenous $\mathrm{H}_{2} \mathrm{O}_{2}$ toward the generation of $\mathrm{O}_{2}$. High-angle annular dark-field scanning transmission electron microscopy (HAADF-STEM) image and the corresponding energy-dispersive X-ray spectroscopy (EDS) elemental mapping (Fig. 3d) further confirm the homogeneous 

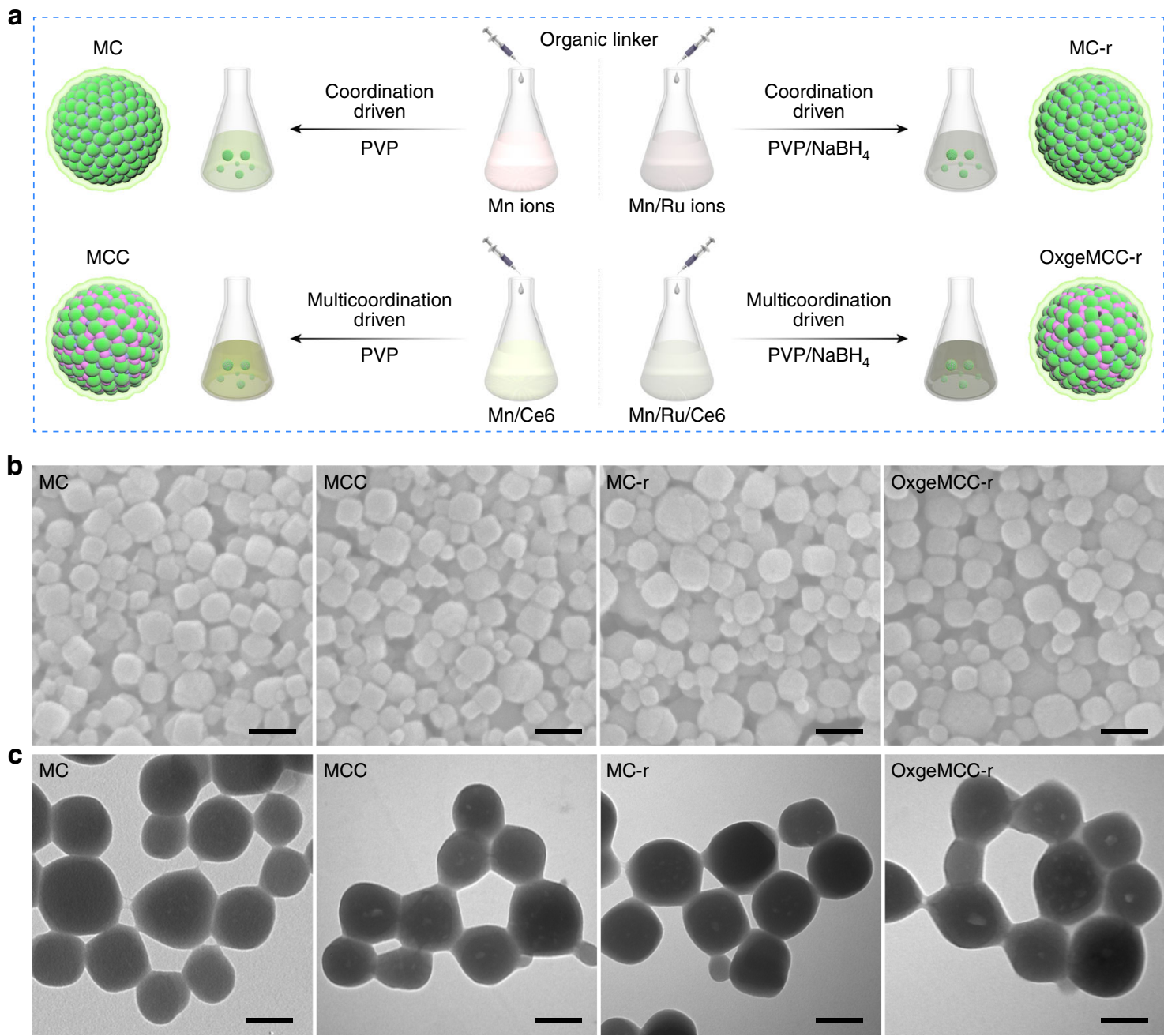

Fig. 2 Synthesis and morphology characterizations. a Schematic illustration for the self-assembly of MC, MCC, MC-r nanoparticles, and OxgeMCC-r SAE. The organic linker $\left[\mathrm{Co}(\mathrm{C} \equiv N)_{6}\right]$ in syringe was added dropwise into four conical flasks filled with different systems under vigorous stirring. Turbidity was observed immediately upon the addition of $\left[\mathrm{Co}(\mathrm{C} \equiv \mathrm{N})_{6}\right]$ in the presence of PVP surfactant. $\mathbf{b}$ SEM images of the as-prepared MC, MCC, MC-r, and OxgeMCC-r. Scale bar is $100 \mathrm{~nm}$. c Corresponding TEM images. Scale bar is $50 \mathrm{~nm}$.

distribution of $\mathrm{Mn}, \mathrm{Co}, \mathrm{Ru}, \mathrm{N}, \mathrm{O}$, and $\mathrm{C}$ elements throughout the OxgeMCC-r SAEs with single-atom Ru content of $2.18 \mathrm{wt} \%$, consistent with the result based on inductively coupled plasma (ICP) measurement (Supplementary Table 1).

X-ray photoelectron spectroscopy (XPS) of MC and MC-r was also performed to identify the existing form of different species. Obviously, Ru $3 p$ peaks at 462.1 and $485.3 \mathrm{eV}$ were assigned to $\mathrm{Ru}$ $3 p_{3 / 2}$ and $\mathrm{Ru} 3 p_{1 / 2}$, respectively (Supplementary Figure 1). As shown in Fig. 3e, an obvious position change for the $\mathrm{C} 1 s$ peak from 284 to $284.4 \mathrm{eV}$ was observed after the Ru doping, confirming that $\mathrm{Ru}$ took the place of some Co sites $^{39}$. A Ru $3 d$ XPS signal was observed but obscured by the C $1 s$ signal at $284.4 \mathrm{eV}$ (inset of Fig. 3e). The deconvoluted spectrum presents one doublet ( $\mathrm{Ru}$ $3 d_{3 / 2}$ at $285.1 \mathrm{eV}$ and $\mathrm{Ru} 3 d_{5 / 2}$ at $281.0 \mathrm{eV}$ ), denoting a main valence state of $\mathrm{Ru}^{0}$ species, which is obviously different from that of pure $\mathrm{RuCl}_{3}$ powder (Supplementary Fig. 2) ${ }^{45}$. To investigate the electronic structure, near-edge X-ray absorption fine structure (NEXAFS) measurements were then performed. For the $\mathrm{C}$ K-edge spectrum of MC (Fig. 3f), peaks $\mathrm{A}(286.8 \mathrm{eV})$ and $\mathrm{B}$ $(288.6 \mathrm{eV})$ are assigned to the excitations of $\mathrm{C} \equiv \mathrm{N} \pi^{*}$ and $\mathrm{C} \equiv \mathrm{N}$ $\sigma^{*} 46,47$. As compared with MC, the positions of peaks $\mathrm{A}$ and $\mathrm{B}$ in MC-r showed slight shifts to lower energy, indicating the changed $2 p$-band electron density of carbon. This phenomenon is originated from the electron donation from electron-rich $\mathrm{Ru}$ single atom to the nearest six carbons, whose $p$-electrons synergistically facilitate the stabilization of $\mathrm{Ru}$ single atom. Furthermore, unchanged positions from $\mathrm{N}$ K-edge spectrum centered at $399.8 \mathrm{eV}$ (peak A) and $401.8 \mathrm{eV}$ (peak B), together with Mn $2 p$ for MC and MC-r, reveal that the $\mathrm{N}_{6}$ coordinative environment is unchanged (Fig. $3 \mathrm{~g}$ and Supplementary Figure 3). Thus, the formation of single-atom $\mathrm{Ru}$ could be simply achieved by the reduction of these noble metal nodes in the presence of low concentration $\mathrm{NaBH}_{4}$ in the solution $^{48,49}$.

As shown in Fig. 4a, the obtained OxgeMCC-r SAEs showed a well monodispersed near-globular morphology with a uniform PVP shell. It should be noted that PVP is a synthetic polymer with good biocompatibility to enhance the stability of OxgeMCCr SAEs in physiological environment ${ }^{50,51}$. The dark region circled with white indicated that $\mathrm{Ce} 6$ was successfully incorporated into the self-assembled system (inset of Fig. 4a). Dynamic light scattering (DLS) measurements suggested relatively larger hydrophilic diameter as compared with the TEM image on account of the PVP coating (Fig. 4b). There was no apparent change in the DLS size within 12 days of storage, and the aqueous dispersion of OxgeMCC-r SAEs remained clear and stable (Supplementary Figure 4). By adjusting the amount of added Ce6 from 0 to $120 \mathrm{mg}$, corresponding loading capacity and loading efficiency varied, and an optimal Ce6 amount of $60 \mathrm{mg}$ 

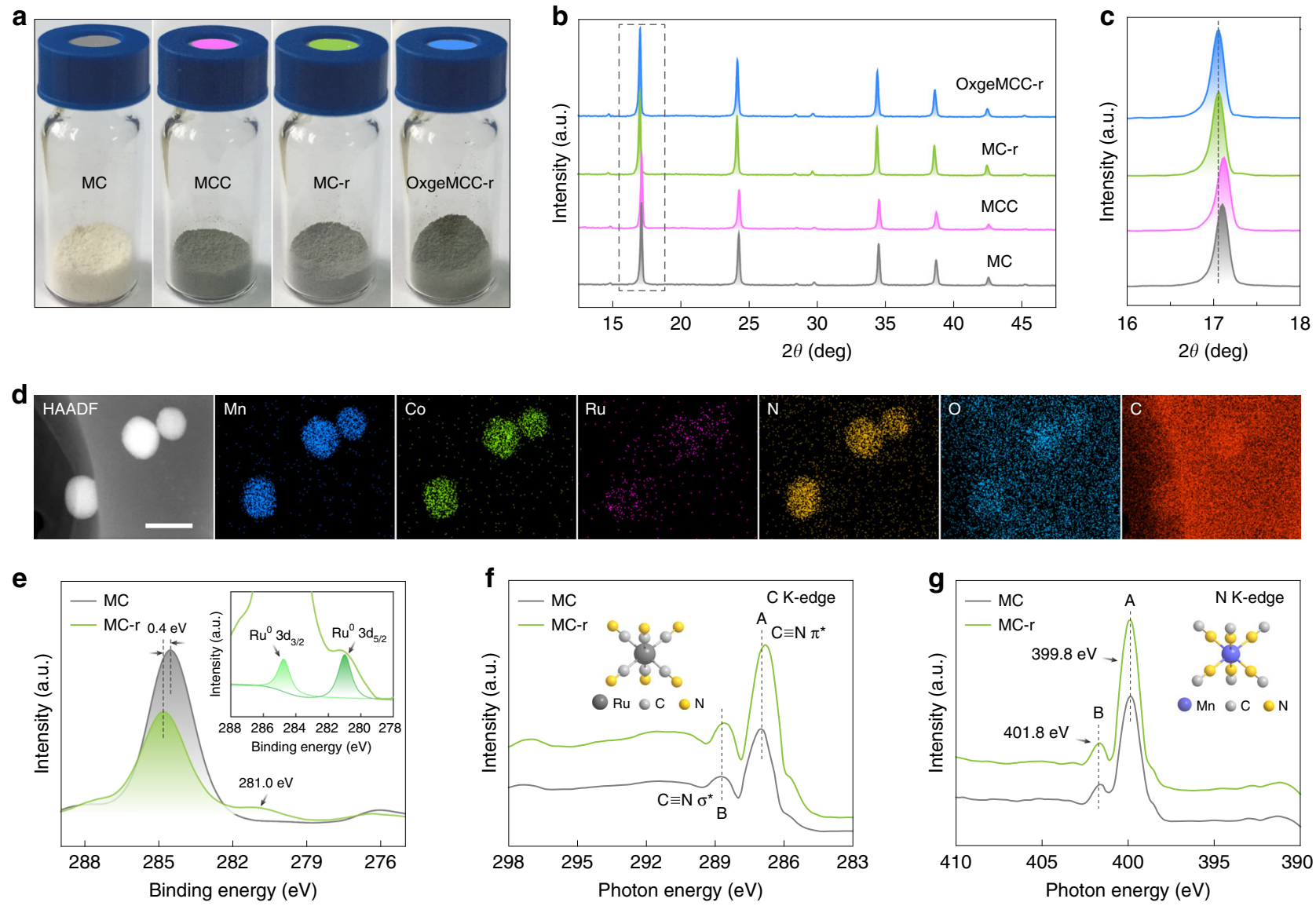

Fig. 3 Structure characterizations. a Photograph of MC, MCC, MC-r, and OxgeMCC-r SAE. b Powder XRD of corresponding materials. c Enlarged powder XRD (2 $\theta$ from 16 to 18 degree) of b. d HAADF-STEM image and corresponding EDS elemental mapping (Mn, Co, Ru, N, O, and C elements) of OxgeMCC-r SAEs. Scale bar is $100 \mathrm{~nm}$. e C 1s XPS spectrum magnified from Supplementary Figs. 1 and 3. Inset is the enlarged Ru 3d spectrum of MC-r. f C K-edge NEXAFS spectra of MC and MC-r. $\mathbf{g}$ N K-edge NEXAFS spectra of MC and MC-r.

was chosen for the following experiments (Supplementary Figure 5). The UV-vis-NIR spectrum confirmed the successful incorporation of Ce6 with high loading capacity of $30.3 \mathrm{wt} \%$ and loading efficiency of $75.8 \%$ (Fig. 4c). The red-shift of the main absorbance peaks for Ce6 in the self-assembled system indicated the interaction between $\mathrm{Mn}$ and $\mathrm{COO}^{-}$group as well as the $\pi-\pi$ stacking interaction between Ce6 and organic linker. Furthermore, $\mathrm{N}_{2}$ adsorption and desorption isotherms were conducted to study the encapsulation of Ce6. As shown in Supplementary Fig. 6, both Brunauer-Emmett-Teller surface area $\left(176.5 \mathrm{~m}^{2} \mathrm{~g}^{-1}\right)$ and pore volume $\left(0.12 \mathrm{~cm}^{3} \mathrm{~g}^{-1}\right)$ of OxgeMCC-r SAEs were significantly lower than that of MC-r $\left(735.8 \mathrm{~m}^{2} \mathrm{~g}^{-1}\right.$ and $0.39 \mathrm{~cm}^{3}$ $\mathrm{g}^{-1}$ ), indicating the occupancy of pores by Ce6 in OxgeMCC-r.

Long-term catalytic ability. After successful synthesis and morphological characterization of OxgeMCC-r SAE, we investigated its capability as a catalyst for converting $\mathrm{H}_{2} \mathrm{O}_{2}$ to $\mathrm{O}_{2}$. In the presence of $\mathrm{H}_{2} \mathrm{O}_{2}(10 \mathrm{mM})$, the $\mathrm{O}_{2}$ generation in the working solution gradually increased upon the addition of MC-r and OxgeMCC-r (Fig. 4d and Supplementary Fig. 7). Conversely, the control group without MC-r and OxgeMCC-r showed no change within the test period. High catalytic ability would reduce the catalyst dosage and promote its further biological applications. The experimental results indicated that, in the presence of MC-r and OxgeMCC-r, $50 \%$ of the added $\mathrm{H}_{2} \mathrm{O}_{2}$ could be consumed within $20 \mathrm{~min}$ (Fig. $4 \mathrm{e}$ and Supplementary Fig. 8). In addition, the catalytic reaction rate constant of OxgeMCC-r was calculated to be $0.041 \mathrm{~min}^{-1}$, which was higher than that of widely used $\mathrm{MnO}_{2}$ $\left(0.029 \mathrm{~min}^{-1}\right)$ under the same active component concentration (0.5 ppm Ru for OxgeMCC-r and 0.5 ppm Mn for $\mathrm{MnO}_{2}$, Supplementary Fig. 9). Thus, the anchored single-atom Ru catalytic site within the self-assembled system would be a superior catalyst toward $\mathrm{H}_{2} \mathrm{O}_{2}$, showing even better catalytic activity than the well-known $\mathrm{MnO}_{2}{ }^{52}$. Furthermore, the catalytic activity of OxgeMCC-r remained unchanged in a mimetic acidic tumor microenvironment $(\mathrm{pH}=6.5)$ after several repeated additions of $\mathrm{H}_{2} \mathrm{O}_{2}$, suggesting its durable catalytic stability (Supplementary Fig. 10). On the other hand, catalytic ability of $\mathrm{MnO}_{2}$ rapidly decreased after two rounds of tests due to acid-induced catalyst self-decomposition (Supplementary Fig. 11). Since achieving continuous $\mathrm{O}_{2}$ supply for long-term hypoxia amelioration is a big challenge that hampers the PDT efficacy in tumor, the asprepared OxgeMCC-r SAEs offer a promising solution to address this issue. Following which, the capability of OxgeMCC-r SAEs for enhanced PDT under a mimetic $\mathrm{H}_{2} \mathrm{O}_{2}$ environment was investigated. The ${ }^{1} \mathrm{O}_{2}$ generation efficiency was measured with 1,3-diphenylisobenzonfuran (DPBF) as ${ }^{1} \mathrm{O}_{2}$ indicator. As shown in Fig. 4f, in hypoxia condition, the ${ }^{1} \mathrm{O}_{2}$ production ability of OxgeMCC-r SAEs under $671 \mathrm{~nm}$ laser irradiation was hampered compared to that in normoxia condition. Interestingly, with addition of $\mathrm{H}_{2} \mathrm{O}_{2}$, a comparable ${ }^{1} \mathrm{O}_{2}$ production was achieved under both hypoxia and normoxia conditions in the presence of OxgeMCC-r SAEs. Considering the mimetic $\mathrm{H}_{2} \mathrm{O}_{2}$ environment and hypoxia condition, the improved ${ }^{1} \mathrm{O}_{2}$ production could be attributed to the amelioration of hypoxic atmosphere through the single-atom based catalysis. 

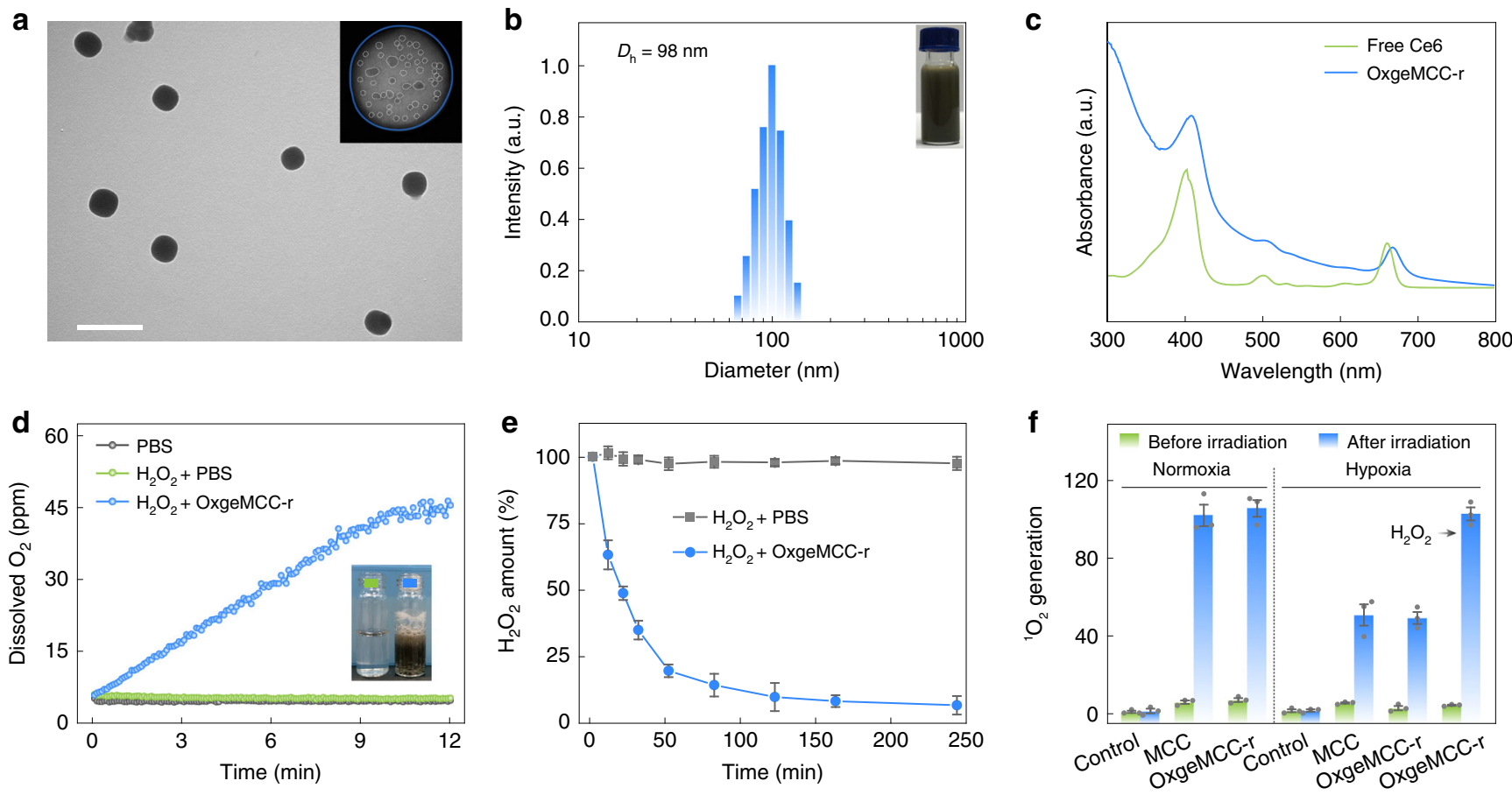

Fig. 4 Structure, oxygen generation, and singlet oxygen generation of OxgeMCC-r SAE. a Representative TEM image. Inset is the enlarged image of one single OxgeMCC-r SAE after reversed-phase treatment. White dotted circles indicate the encapsulated Ce6. Scale bar is $200 \mathrm{~nm}$. b DLS profile with the inset picture of the sample dispersed in water. c UV-vis absorption spectra of free Ce6 and OxgeMCC-r SAE. d $\mathrm{O}_{2}$ generation after treating OxgeMCC-r SAE with $\mathrm{H}_{2} \mathrm{O}_{2}$ in PBS. Inset is a photograph of $\mathrm{H}_{2} \mathrm{O}_{2}$ solutions in the presence or absence of OxgeMCC-r SAE. e Degradation profiles of $\mathrm{H}_{2} \mathrm{O}_{2}$ with or without of OxgeMCC-r SAE. $\mathbf{f}$ Singlet oxygen $\left({ }^{1} \mathrm{O}_{2}\right)$ generation ability determined by DPBF indicator under different conditions before and after laser irradiation $\left(671 \mathrm{~nm}, 100 \mathrm{~mW} \mathrm{~cm}^{-2}, 30 \mathrm{~s}\right)$. Data are presented as mean \pm s.e.m. $(n=3)$.

Intracellular oxygen generation. On the basis of above results, endogenous $\mathrm{H}_{2} \mathrm{O}_{2}$ decomposition and intracellular oxygen generation of MC-r were investigated subsequently. The cytotoxicity was first studied before conducting further biological applications. More than $88 \%$ of $4 \mathrm{~T} 1$ cells survived when incubated with MC-r at a concentration as high as $200 \mathrm{ppm}$ (Fig. 5a), indicating satisfactory biocompatibility of the obtained MC-r nanoparticles. It is well established that hypoxia condition induces intracellular expression of HIF-1a protein ${ }^{53}$. We then wanted to understand how the level of HIF-1 $\alpha$ in hypoxic cells changes after incubation with MC-r. In a typical experiment, HIF-1a protein and tubulin were stained using anti-HIF- $1 \alpha$ antibody and anti- $\alpha$-Tubulin antibody, respectively. As shown in Fig. 5b, the immunofluorescence imaging results showed no obvious expression of HIF-1a under normoxia condition. In contrast, when incubated under hypoxia condition, enhanced expression of HIF-1a can be observed with the brightest green fluorescence. However, upon treating with MC-r, the expression of HIF-1a was significantly down-regulated with the increase of MC-r concentration from 25 to $50 \mathrm{ppm}$ (Fig. $5 \mathrm{c}$ ), suggesting that efficient oxygenation induced by MC-r attenuated the hypoxic condition. Almost no green fluorescence signal was observed when using rabbit IgG as the isotype control, demonstrating the specific binding of HIF-1a antibody (Supplementary Fig. 12). Furthermore, Western blot analysis was employed to study the expression of HIF-1a protein under various conditions. The Western blot data showed a similar tendency as compared with immunofluorescence results (Fig. 5d and Supplementary Fig. 13). All groups exhibited a similar level of tubulin amount, indicating high hypoxia amelioration ability of MC-r without disturbing the cytoskeleton. The intracellular $\mathrm{O}_{2}$ generation capacity was also studied using an intracellular $\mathrm{O}_{2}$ level indicator $\left[\mathrm{Ru}(\mathrm{dpp})_{3}\right] \mathrm{Cl}_{2}$. As shown in Fig. 5e, under hypoxia condition, the group treated with MC-r showed much weaker fluorescence compared to the hypoxia control group, indicating significant intracellular $\mathrm{O}_{2}$ supply by $\mathrm{MC}-\mathrm{r}$ via the singleatom based catalysis. Notably, results also demonstrated a concentration-dependent intracellular $\mathrm{O}_{2}$ generation ability of MC-r and hence the hypoxia condition could be easily modulated. These phenomena collectively validated the ability of MC-r to conduct in situ $\mathrm{H}_{2} \mathrm{O}_{2}$ decomposition and intracellular $\mathrm{O}_{2}$ generation.

Enhanced in vitro PDT. Encouraged by the intracellular $\mathrm{O}_{2}$ generation, the enhanced PDT efficiency of OxgeMCC-r SAE was assessed against 4T1 cell line. Before which, the subcellular localization of MCC and OxgeMCC-r SAE were studied by confocal laser scanning microscopy. Results indicated that both MCC and OxgeMCC-r SAE localized in endosomes/lysosomes (Supplementary Fig. 14). Furthermore, cell viability results demonstrated that MC-r, MCC, and OxgeMCC-r showed negligible cytotoxicity to 4T1, HeLa, and non-cancerous HEK 293 cell lines under the tested concentrations in the dark condition (Supplementary Figs. 15 and 16). When applied NIR irradiation, both MCC and OxgeMCC-r groups under normoxic condition exhibited comparable phototoxicity toward $4 \mathrm{~T} 1$ cells at a wide range of Ce6 concentrations using standard 3-(4,5-dimethylthiazol-2-yl)-2,5-diphenyltetrazolium bromide (MTT) method (Fig. 6a). That is to say, the $\mathrm{O}_{2}$ supply is sufficient for these tested groups under normoxic condition. It should be noted that higher phototoxicity for MCC and OxgeMCC-r groups as compared with free $\mathrm{Ce} 6$ group was due to the enhanced intracellular delivery of $\mathrm{Ce} 6$ with the assistance of the self-assembled system. As the hypoxia condition is an intrinsic property of the solid tumor, a mimetic hypoxic condition was achieved through culturing cancer cells within an incubator furnished with hypoxic 

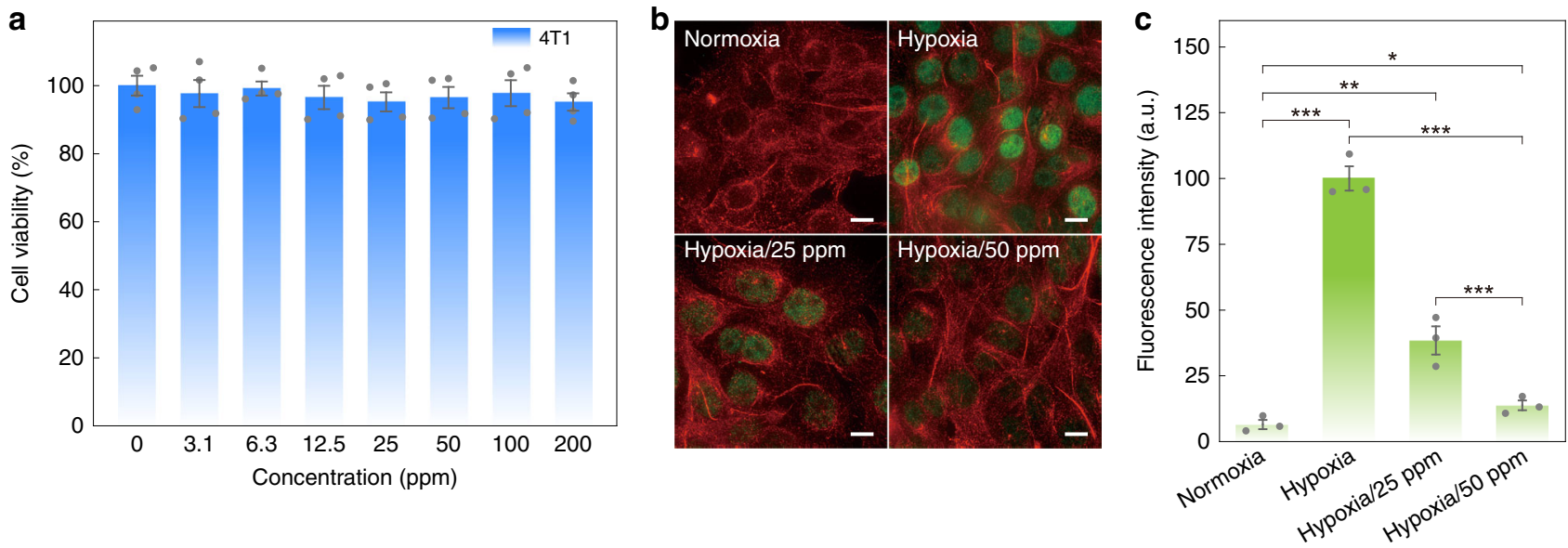

d
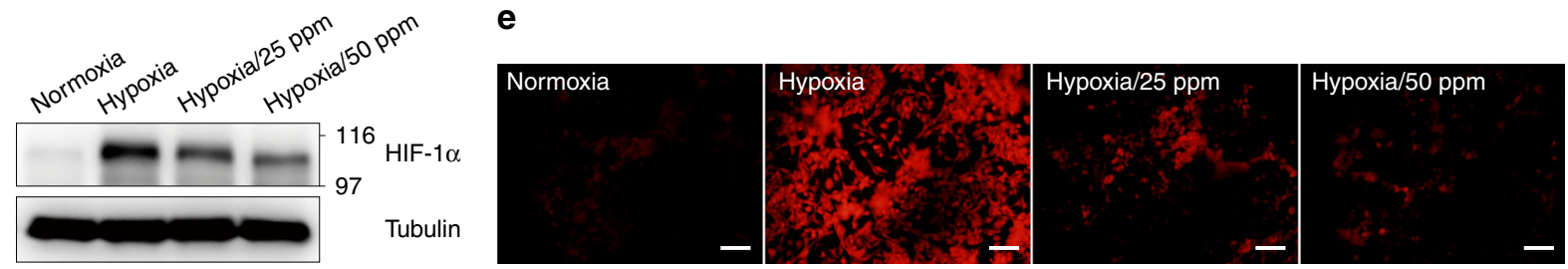

Fig. 5 In vitro biocompatibility, alleviation of hypoxic condition, and intracellular $\mathbf{O}_{\mathbf{2}}$ generation. a $4 \mathrm{~T} 1$ cells viability after treated with $\mathrm{MC}$ - $\mathrm{r}$ nanoparticles with different concentrations. Data are presented as mean \pm s.e.m. $(n=4)$. b Fluorescence imaging of $4 \mathrm{~T} 1$ cells with stained HIF- $1 \alpha$ (green) and tubulin (red) after treated with PBS under normoxic condition $\left(21 \% \mathrm{O}_{2}, 5 \% \mathrm{CO}_{2}\right.$, and $74 \% \mathrm{~N}_{2}$ ) as well as PBS (Hypoxia) and MC-r (Hypoxia/25 ppm and Hypoxia/50 ppm) under hypoxic condition $\left(1 \% \mathrm{O}_{2}, 5 \% \mathrm{CO}_{2}\right.$, and $\left.94 \% \mathrm{~N}_{2}\right)$. Scale bar is $10 \mu \mathrm{m}$. c Relative intensity of corresponding green fluorescence from HIF-1 $\alpha$ under different treating conditions. Data are presented as mean \pm s.e.m. $(n=3)$. Statistical analysis was performed via one-way ANOVA. ${ }^{\star} p<$ $0.05,{ }^{\star \star} p<0.01,{ }^{\star \star \star} p<0.001$. d Western blot analysis of HIF-1 $\alpha$ expression in $4 \mathrm{~T} 1$ cells under different treating conditions. e Fluorescence imaging of $4 \mathrm{T1}$ cells stained by $\mathrm{O}_{2}$ indicator after treatments with different conditions. Scale bar is $100 \mu \mathrm{m}$.

atmosphere $\left(\mathrm{N}_{2} / \mathrm{CO}_{2} / \mathrm{O}_{2}: 94 / 5 / 1\right.$ in volume ratio) for $2 \mathrm{~h}$ before treatment. Under hypoxic condition, the PDT cytotoxicity of free Ce6 and MCC groups was only $18.4 \%$ and $25.3 \%$ respectively, due to insufficient $\mathrm{O}_{2}$ supply (Fig. 6b). Remarkably, when incubated with OxgeMCC-r (26.7 ppm, containing 8 ppm of Ce6 and 18.7 ppm of MC-r according to the weight ratio) followed by $671 \mathrm{~nm}$ laser irradiation, nearly $90 \%$ of cancer cells were killed. The augmented PDT efficacy was accounted to sufficient $\mathrm{O}_{2}$ supply generated via the single-atom based catalysis with overexpressed intracellular $\mathrm{H}_{2} \mathrm{O}_{2}{ }^{54}$. Considering the limited mitochondrial activity under hypoxic condition that may affect the MTT results, another independent CellTiter-Fluor assay that measures the protease activity within live cells was also conducted. As shown in Supplementary Fig. 17, the in vitro PDT results were basically consistent with the MTT data.

The in vitro tumor cell killing efficiency under the mimetic hypoxic condition was further confirmed by calcein-AM (green, live cells) and propidium iodide (red, dead cells) double-staining. With the treatment of free Ce6 or MCC under hypoxia condition, relatively low red fluorescence was observed (Fig. 6c), suggesting inadequate PDT efficiency. In contrast, OxgeMCC-r group presented the highest percentage of dead cells, which was consistent with the results from the MTT assay results. Furthermore, the lethal mechanism was investigated through flow cytometry analysis. Compared to control group, both free Ce6 and MCC groups showed small migration from Q4 (live cells) to Q2 (early apoptosis) and Q3 (late apoptosis), indicating the apoptosis-induced cell death pathway (Fig. 6d). In contrast, the apoptosis percentage of the OxgeMCC-r group presented significantly high value of $72.4 \%$ than that of Ce6 group (8.2\%) and MCC group (26.8\%). In addition, intracellular ROS generation was measured using $2^{\prime}, 7^{\prime}$-dichlorodihydrofluorescein diacetate $\left(\mathrm{H}_{2}\right.$-DCFDA) as a ROS detector. Under hypoxic condition, 4T1 cells treated with OxgeMCC-r SAEs exhibited an enhanced ROS stress level as compared with group treated with MCC nanoparticles (Supplementary Fig. 18). All the above results demonstrated that OxgeMCC-r SAEs could ameliorate hypoxia condition and play an important role in sustaining dependable PDT efficiency.

MR imaging ability. In light of excellent results from above in vitro studies, OxgeMCC-r SAE was applied on animal models in vivo. Due to the fact that $\mathrm{Mn}$ is coordinated to six nitrogen atoms forming high-spin $\mathrm{Mn}-\mathrm{N}_{6}(\mathrm{~S}=5 / 2)$ species, it is no doubt that OxgeMCC-r SAE can be a suitable agent for MR imaging. Thus, we investigated the in vivo MR imaging performance of OxgeMCC-r SAE on a subcutaneous tumor model (Fig. 7a). For diagnostic purpose, we first tested the MR imaging functions of OxgeMCC-r $\mathrm{SAE}$ in physiological condition. The $\mathrm{T}_{1}$-weighted magnetic resonance images of OxgeMCC-r obtained with a 3.0-T magnetic resonance clinical scanner (GE HDxt) demonstrated a concentration-dependent signal enhancement effect (Fig. 7b, c). The corresponding longitudinal relaxivity $\left(r_{1}\right)$ value was quantitatively calculated to be $5.44 \mathrm{mM}^{-1} \mathrm{~s}^{-1}$, which was higher than clinically Gd-based contrast agent (Magnevist, $\left.r_{1}=4.56 \mathrm{mM}^{-1} \mathrm{~s}^{-1}\right)^{55}$. Thereafter, time-dependent MR imaging ability in 4T1 cells incubated with OxgeMCC-r was assessed. Upon increasing the incubation time, brighter imaging intensity was observed on account of increased cell endocytosis (Fig. 7d). The quantification results demonstrated that OxgeMCC-r SAE after the incubation for $6 \mathrm{~h}$ had a significantly higher value than that of $2 \mathrm{~h}$ (Fig. 7e). More importantly, in vivo positive signal enhancement within $48 \mathrm{~h}$ postinjection suggested that the OxgeMCC-r SAE could be accumulated in the tumor site through the enhanced permeability and retention (EPR) effect $^{56}$. Simultaneously, MR imaging signals were detected 
a

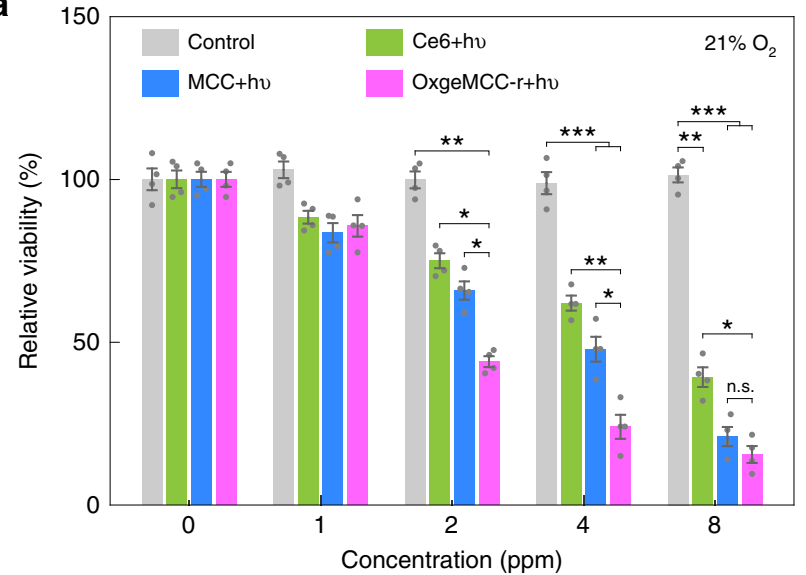

C
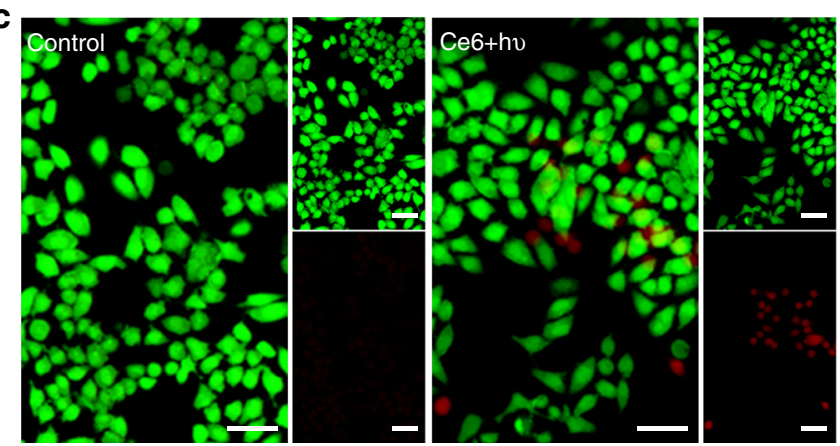

b

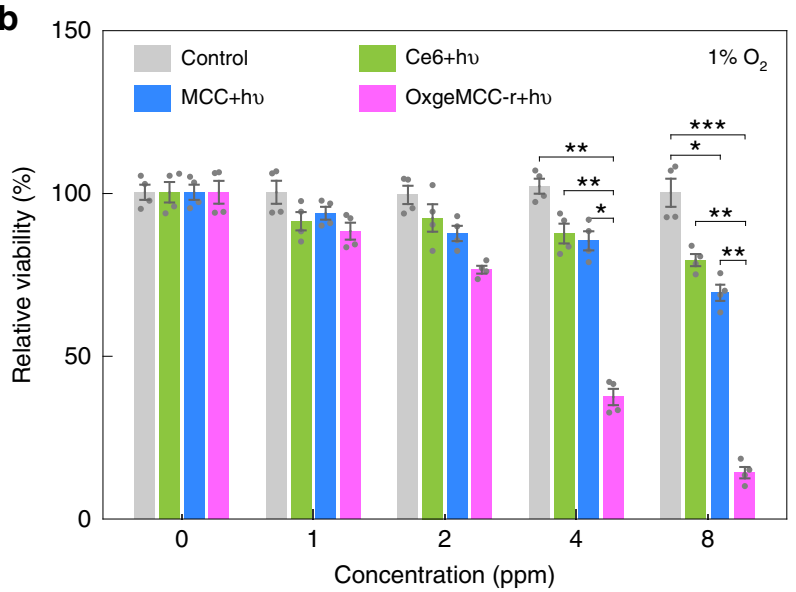

d
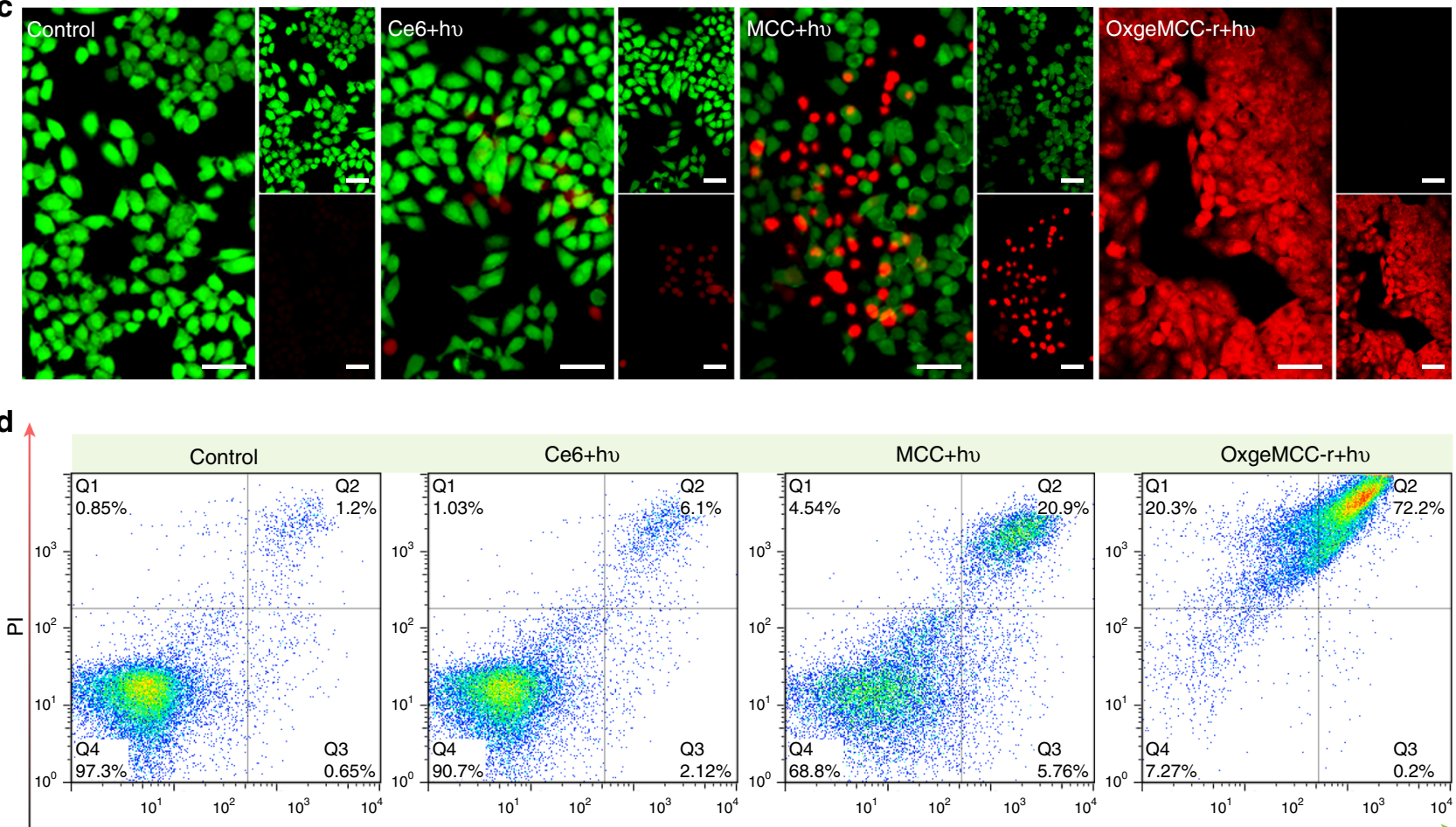

Annexin-V FITC

Fig. 6 In vitro PDT evaluation of OxgeMCC-r SAE on 4T1 tumor cells. Cell viability assay of free Ce6, MCC, and OxgeMCC-r SAE treated 4T1 cells in a normoxic and $\mathbf{b}$ hypoxic conditions under $671 \mathrm{~nm}$ light irradiation (concentration of Ce6: 8 ppm; $671 \mathrm{~nm}$ laser power density: $100 \mathrm{~mW}$ cm ${ }^{-2}$; irradiation time: $30 \mathrm{~s}$ ). Data are presented as mean \pm s.e.m. $(n=4)$. Statistical analysis was performed via one-way ANOVA. ${ }^{\star} p<0.05,{ }^{\star \star} p<0.01,{ }^{\star \star \star} p<0.001$. c Live/dead staining of 411 cells treated with PBS, free Ce6, MCC, and OxgeMCC-r SAE in the presence of 671 nm laser irradiation under hypoxic conditions. Green signal from calcein AM indicates live cells and red signal from propidium iodide (PI) indicates dead cells (concentration of Ce6: 8 ppm). Scale bar is $50 \mu \mathrm{m}$. d Cell death mechanism after the treatment of PBS, free Ce6, MCC, and OxgeMCC-r SAE in the presence of $671 \mathrm{~nm}$ laser irradiation under hypoxic conditions assessed with Annexin V-FITC/PI by flow cytometry.

in the tumor region, reaching the maximum after $6 \mathrm{~h}$ of intravenous injection through the tail vein, suggesting time-dependent tumor accumulation behavior of OxgeMCC-r SAEs (Fig. 7f). Quantitative MR imaging signals within the tumor site treated by OxgeMCC-r SAE further confirmed the findings (Fig. 7g). The long-lasting imaging ability of the OxgeMCC-r SAE would be very useful for guiding in vivo therapy.

In vivo biocompatibility. The biocompatibility of OxgeMCC-r SAE was further investigated. The hemolysis tests were performed to study the biocompatibility of OxgeMCC-r SAE in blood (Supplementary Fig. 19). Results showed that the hemolysis percentage was less than $2 \%$ even at an incubation concentration as high as $1000 \mathrm{ppm}$. Next, histological examination of various major organs (heart, kidney, liver, lung, spleen, and intestine) from mice injected with OxgeMCC-r SAEs and PBS (as control) was conducted to investigate the potential biological toxicity (Fig. 7h). There was no obvious pathological abnormality or inflammation observed from the mice for both groups. In addition, blood biochemical and blood routine analysis were also performed to understand long-term biosafety of OxgeMCC-r SAE. The blood was collected at different time points after the administration of OxgeMCC-r SAEs. For blood biochemistry, all functional markers, including blood urea nitrogen (BUN), alkaline phosphatase (ALP), alanine transaminase (ALT), and 
a

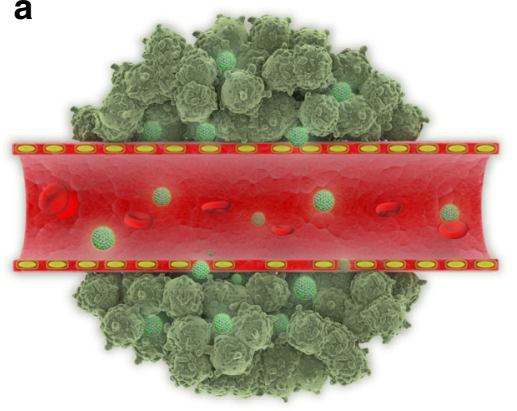

b

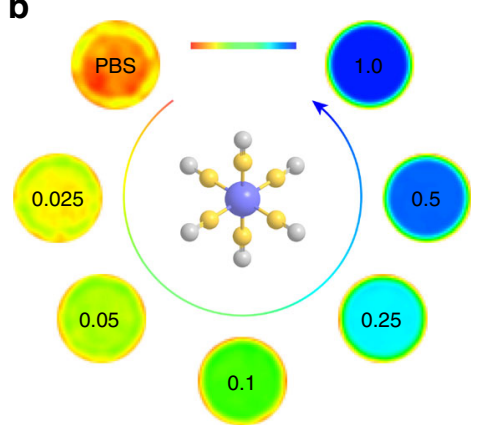

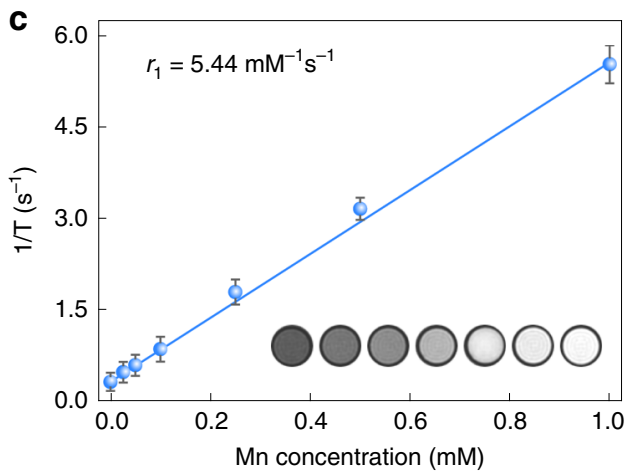
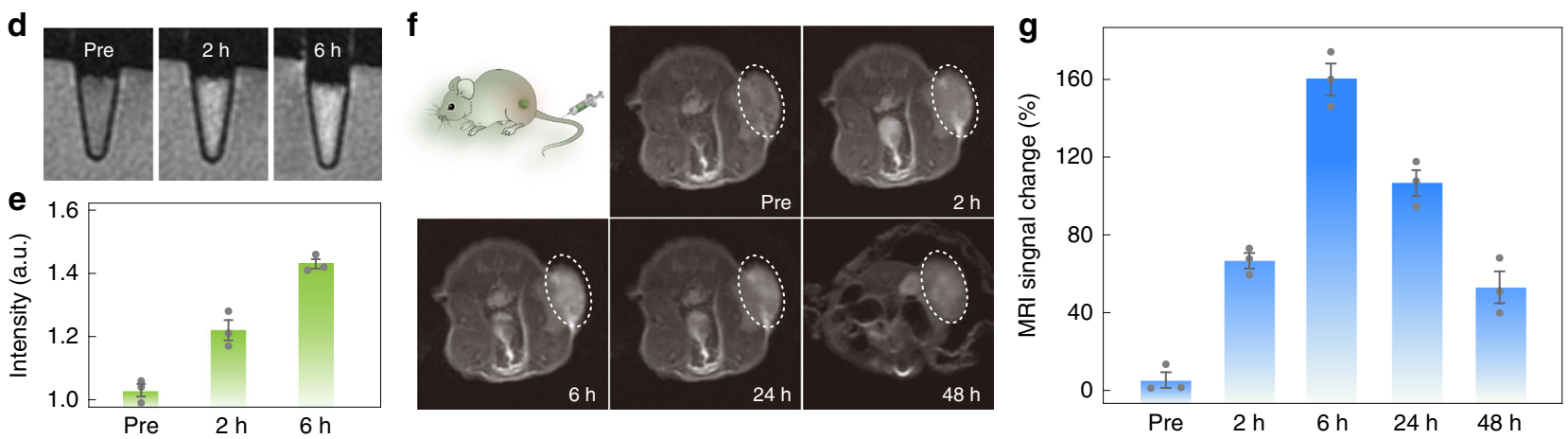

h
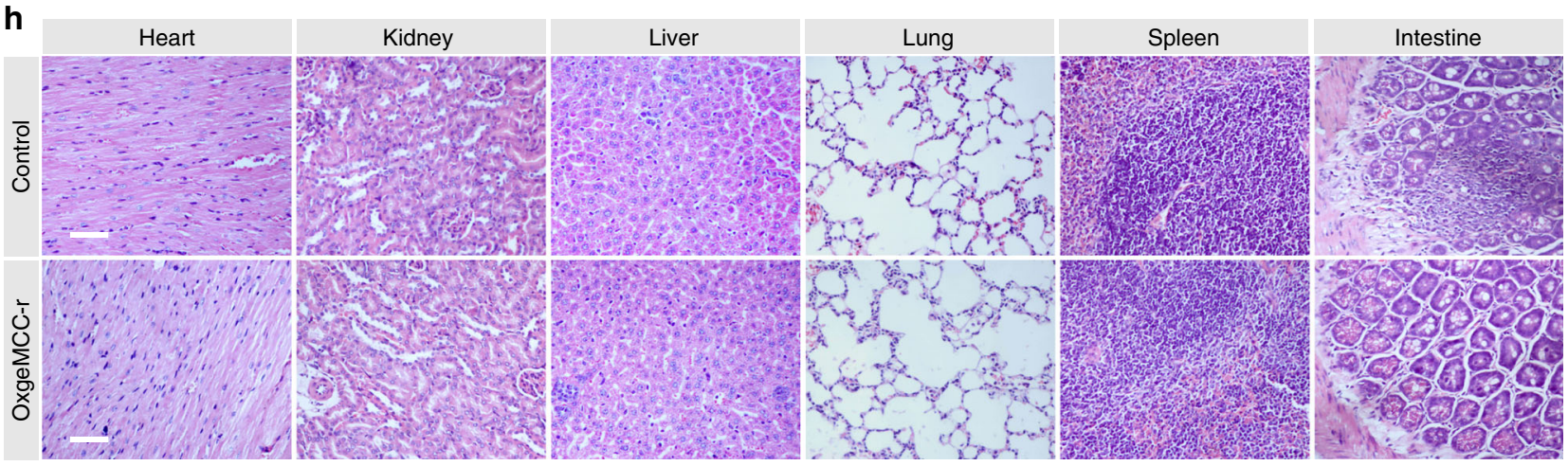

Fig. 7 In vitro/vivo MR imaging and biocompatibility of OxgeMCC-r SAE. a Accumulation of OxgeMCC-r SAE at the tumor site through the EPR effect. b In vitro $T_{1}$-weighted magnetic resonance images of OxgeMCC-r SAE in aqueous solution with various Mn concentrations (mM). c Transverse relativity $\left(r_{1}\right)$ value of $5.44 \mathrm{mM}^{-1} \mathrm{~s}^{-1}$ for OxgeMCC-r SAE. Inset is magnetic resonance phantom images of OxgeMCC-r SAE. Data are presented as mean \pm s.e.m. $(n=3)$. $\mathbf{d} \mathrm{T}_{1}$-weighted MR imaging of $4 \mathrm{T1}$ cells treated with PBS, OxgeMCC-r SAE for $2 \mathrm{~h}$, and OxgeMCC-r SAE for $6 \mathrm{~h}$. e Corresponding relative MR imaging intensity of (d). Data are presented as mean \pm s.e.m. $(n=3)$. $\mathbf{f}$ In vivo $\mathrm{T}_{1}$-weighted magnetic resonance images of $4 \mathrm{~T} 1$ tumor-bearing mouse at various time points post-injection. Tumor regions are marked with white dashed lines. $\mathbf{g}$ Quantitative $\mathrm{T}_{1}$-weighted MR imaging signals within the tumor site. Data are presented as mean \pm s.e.m. $(n=3)$. h Micrographs of major organs stained with H\&E. Scale bar is $50 \mu \mathrm{m}$.

aspartate aminotransferase (AST) were measured. The aminotransferase levels at days 8 and 16 showed no apparent change for the tested groups, indicating compatible kidney and hepatic property of OxgeMCC-r SAEs (Supplementary Table 2). For blood routine analysis, results revealed the tested nine common indexes are within the normal ranges, indicating negligible blood toxicity of OxgeMCC-r SAE under the treatment dose within 16 days in vivo (Supplementary Table 3$)^{57}$. Overall, these results firmly demonstrated the in vivo biocompatibility of OxgeMCC-r SAE, potentiating its further application as a theranostic agent.

In vivo PDT. Encouraged by the superior in vitro PDT efficacy, satisfactory biocompatibility as well as high tumor accumulation, in vivo PDT of OxgeMCC-r SAE was carried out on 4T1 tumorbearing mice. The mice were randomly divided into four groups (control, free Ce6, MCC, and OxgeMCC-r, $n=5$ ). For mice treated with light irradiation, the animals were subjected to irradiation ( $671 \mathrm{~nm}$ laser, $100 \mathrm{~mW} \mathrm{~cm}^{-2}, 5 \mathrm{~min}$ ) at $6 \mathrm{~h}$ post intravenous injection. Our results clearly showed the control group exhibited the fastest tumor growth (Fig. 8a). Similar results were also observed for free Ce6 group with no noticeable tumor growth inhibition under laser irradiation due to fast metabolism of the free drug form. The tumor growth in mice administrated with MCC could be partially inhibited on account of the efficient delivery of $\mathrm{Ce} 6$ to tumor sites (Fig. 8b). Excitingly, the group treated with OxgeMCC-r SAEs showed remarkable tumor suppression under same dosage of laser irradiation. During the treatments, mice from all groups showed the same increasing trend in body weights and no obvious changes in the food and water intake, indicating superior safety of $\mathrm{MC}$ and $\mathrm{MC}-\mathrm{r}$ for delivering theranostic agents in vivo (Supplementary Fig. 20). At the end of treatment (day 14), mice from all groups were euthanized and tumor tissues were harvested. Results revealed the 
a

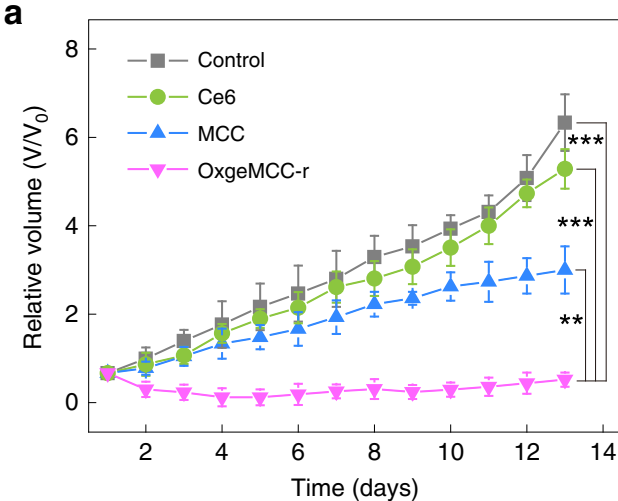

C

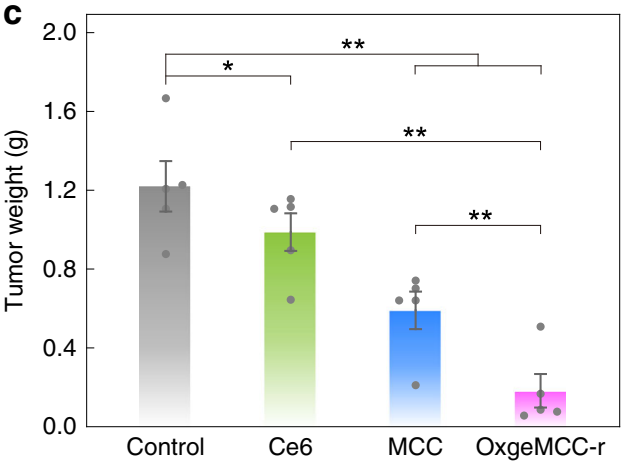

b

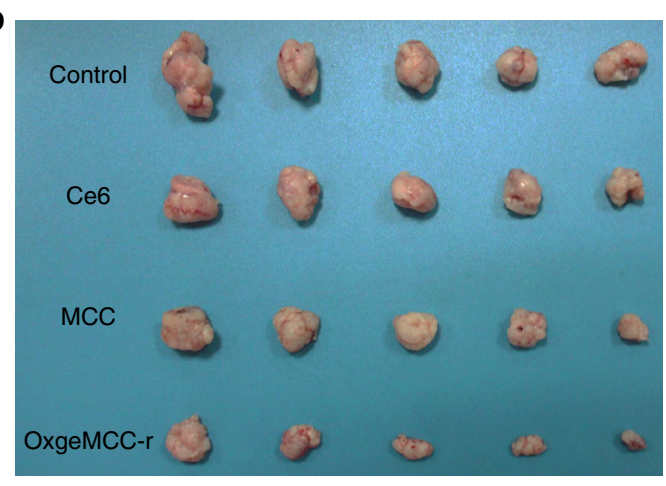

d

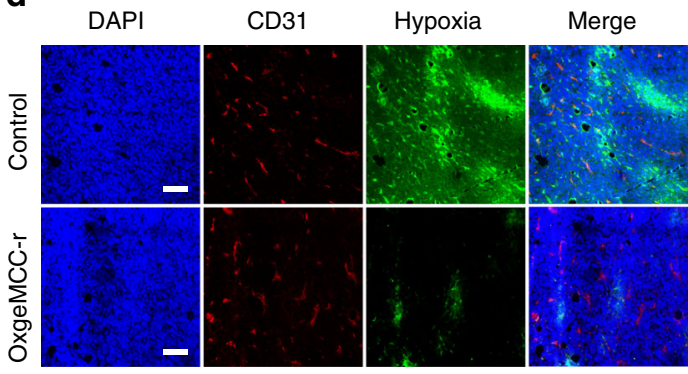

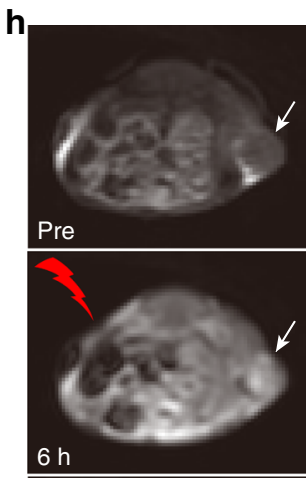

$6 \mathrm{~h}$

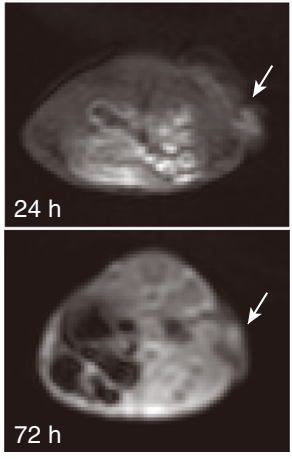

e

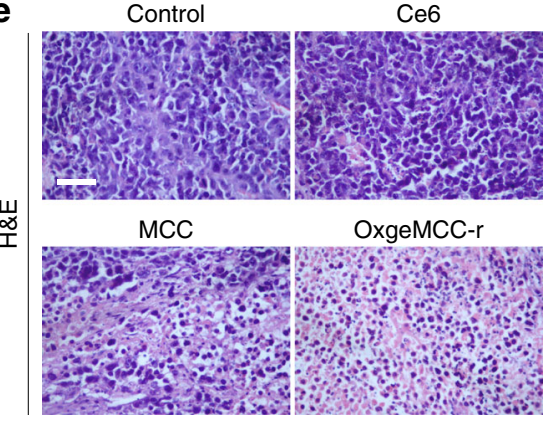

f

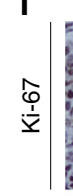

g

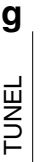

Control

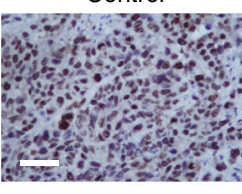

Control

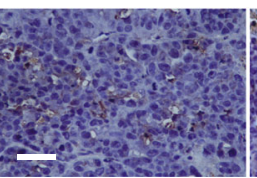

Ce6

MCC
OxgeMCC-r
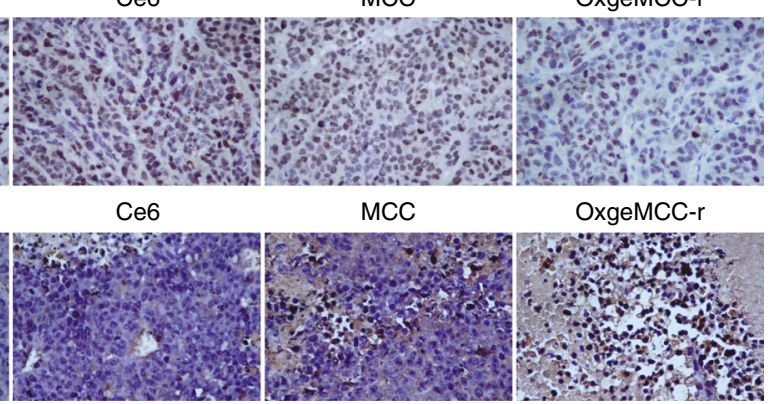

MCC

OxgeMCC-r

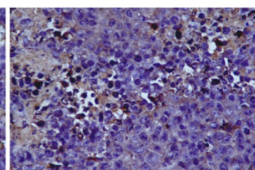

Q

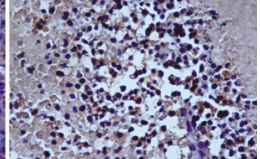

Fig. 8 In vivo PDT efficiency assessments. a Relative tumor volumes of mice after various treatments (control, Ce6, MCC, and OxgeMCC-r, $n=5$ ). The last three groups were treated with laser irradiation: $5 \mathrm{~min}$ with a power density of $100 \mathrm{~mW} \mathrm{~cm}{ }^{-2}$. Injection dose is $100 \mu \mathrm{L}$, with a Ce6 concentration of 4 $\mathrm{mg} \mathrm{kg}^{-1}$. b Photographic images of tumors excised from different groups after various treatments indicated. c Average weights of tumors from different groups of mice after various treatments indicated. d Representative hypoxia immunofluorescence images of tumor slices. Nuclei, blood vessels, and hypoxic regions were stained by 4',6-diamidino-2-phenylindole (DAPI, blue), anti-CD31 antibody (red), and anti-pimonidazole antibody (green), respectively. Scale bar is $100 \mu \mathrm{m}$. e H\&E staining and $\mathbf{f} \mathrm{Ki}-67$ staining of tumor slices from different groups after various treatments. Scale bar is $50 \mu \mathrm{m}$. $\mathbf{g}$ TUNEL staining of tumor slices from different groups indicated. Scale bar is $50 \mu \mathrm{m}$. $\mathbf{h}$ MR imaging of tumor-bearing mouse at different treatment points. Data are presented as mean \pm s.e.m. $(n=5)$. Statistical analysis was performed via one-way ANOVA. ${ }^{\star} p<0.05,{ }^{\star \star} p<0.01,{ }^{\star \star \star} p<0.001$.

average weight of tumor tissues obtained by anatomy for OxgeMCC-r group was the lowest, at only $0.19 \mathrm{~g}$ (Fig. 8c). In comparison to the MCC group, the therapeutic efficacy achieved by OxgeMCC-r SAE group was exceptional, demonstrating a synergistic effect owing to the increase in the localized availability of $\mathrm{O}_{2}$ for self-sustained photodynamic therapy.

The immunofluorescence staining was performed to further confirm the capability of OxgeMCC-r SAE for in situ amelioration of hypoxia status within tumor. For doing this, pimonidazole hydrochloride was first employed as a hypoxia probe to test the hypoxia status of tumor tissues ${ }^{52}$. Tumor hypoxia and blood vessels were then stained with anti-pimonidazole antibody (green signals) and anti-CD31 antibody (red signals), respectively. Results suggested group treated with OxgeMCC-r SAEs displayed significantly reduced hypoxia signals. In contrast, the control group exhibited bright green hypoxia signals (Fig. 8d). It could be concluded that tumor hypoxia condition was alleviated due to the catalysis of $\mathrm{H}_{2} \mathrm{O}_{2}$ into $\mathrm{O}_{2}$ in the presence of OxgeMCC-r SAEs. Next, we performed hematoxylin and eosin (H\&E) staining of tumor slices from different groups to assess the therapeutic efficiency (Fig. 8e). Among all the conducted groups, mice treated with OxgeMCC-r SAEs under laser irradiation suffered the most prominent tumor tissue damage as noticed, validating its high PDT effect. Furthermore, Ki-67 and TUNEL staining were conducted on tumor slices to study the tumor cell proliferation and apoptosis levels, respectively. The OxgeMCC-r SAE group exhibited the least brown area, indicating its excellent inhibiting effect on the proliferation of tumor cells (Fig. 8f). As expected, the highest level of positive TUNEL staining signals on tumor slices was detected from the OxgeMCC-r SAE group, which was consistent with the $\mathrm{H} \& \mathrm{E}$ and $\mathrm{Ki}-67$ staining results (Fig. $8 \mathrm{~g})^{58}$. 
In vivo MR imaging-guided PDT. As a proof of concept for being a theranostic nanoplatform, we performed MR imaging for real-time observation of the tumor growth on $4 \mathrm{~T} 1$ tumor-bearing mouse after administration of OxgeMCC-r SAEs followed by PDT. After the accumulation in the tumor sites, OxgeMCC-r could effectively integrate tumor PDT and prolonged MR imaging in a spatiotemporal manner. As shown in Fig. 8h, magnetic resonance images showed significant OxgeMCC-r accumulation within the tumor site (indicated by the white arrow) at $6 \mathrm{~h}$ post injection. After which, the tumor sites received $671 \mathrm{~nm}$ laser irradiation. Noticeable shrinkage of tumor volume was observed $24 \mathrm{~h}$ after injection (18 h after PDT). Furthermore, almost complete tumor elimination at 3 days after the PDT treatment was observed through MR imaging results. Thus, the developed OxgeMCC-r SAE could be a potential theranostic nanoplatform for MR imaging guided tumor PDT.

\section{Discussion}

Catalysis-based nanomedicine has received much research attention, since this approach shows superior therapeutic efficacy in the cancer treatment. By using MOFs as single-atom anchoring materials, we have successfully developed a multifunctional OxgeMCC-r single-atom nanozyme through one-step selfassembly strategy. OxgeMCC-r SAE with $2.23 \mathrm{wt} \%$ single-atom $\mathrm{Ru}$ loading content was capable of degrading intracellular $\mathrm{H}_{2} \mathrm{O}_{2}$ to $\mathrm{O}_{2}$ to relieve the hypoxia condition of solid tumor, leading to an enhanced ROS generation, and finally causing apoptotic cell death both in vitro and in vivo. Unlike traditional drug carriers, OxgeMCC-r SAE presents a high loading capacity of Ce6 photosensitizer. OxgeMCC-r SAE could selectively accumulate within tumor sites for enhanced PDT of cancer under the guide of $\mathrm{T}_{1}$ MR imaging. Having high loading capacity for $\mathrm{Ce} 6$, superior catalytic property, and suitable imaging capability for in vivo tracking, the OxgeMCC-r SAE would be a promising anticancer theranostic agent, advancing further development of different single-atom nanozymes in the field of catalytic nanomedicine.

\section{Methods}

Preparation of OxgeMCC-r SAE. For the synthesis of OxgeMCC-r SAEs, $\mathrm{K}_{3}[\mathrm{Co}$ $(\mathrm{CN})_{6}$ ] powder $(166 \mathrm{mg})$ was dispersed in ultrapure water $(20 \mathrm{~mL})$ with a final concentration of $8.3 \mathrm{mg} / \mathrm{mL}$. $\mathrm{RuCl}_{3}$ solution $(10 \mathrm{mM})$ was also prepared using ultrapure water. The hydrophobic drug Ce6 $(60 \mathrm{mg})$ was dissolved in ethanol (15 $\mathrm{mL})$. Then, $\mathrm{Mn}\left(\mathrm{CH}_{3} \mathrm{COO}\right)_{2} \cdot 4 \mathrm{H}_{2} \mathrm{O}(25 \mathrm{mM}, 30 \mathrm{~mL})$ aqueous solution, $\mathrm{RuCl}_{3}$ aqueous solution $(5 \mathrm{~mL})$ and $\mathrm{Ce} 6(15 \mathrm{~mL})$ ethanol solution were mixed to form a homogeneous solution. Using PVP $(5 \mathrm{mg} / \mathrm{mL})$ as the surfactant, $\mathrm{K}_{3}\left[\mathrm{Co}(\mathrm{CN})_{6}\right]$ solution $(20 \mathrm{~mL})$ was dropwise added to the mixed solution using a syringe with the total volume reaching about $70 \mathrm{~mL}$ under magnetic stirring. Turbidity generated immediately upon the addition of $\mathrm{K}_{3}\left[\mathrm{Co}(\mathrm{CN})_{6}\right]$ solution. The final concentration of $\mathrm{Mn}(\mathrm{II}), \mathrm{Ce} 6, \mathrm{Ru}(\mathrm{III}), \mathrm{K}_{3}\left[\mathrm{Co}(\mathrm{CN})_{6}\right]$, and PVP was $0.59,0.86,0.072$, 2.37 , and $5 \mathrm{mg} \mathrm{mL}^{-1}$, respectively. By mixing the above solution with $\mathrm{NaBH}_{4}(10$ $\mathrm{mL}, 1 \mathrm{mg} / \mathrm{mL}$ ) solution and stirring for another $12 \mathrm{~h}$, the OxgeMCC-r SAE was obtained. The fabrication of MC-r nanoparticles was achieved without the addition of Ce6. The synthesis of MCC nanoparticles was achieved without the addition of $\mathrm{Ru}(\mathrm{III})$, and for MC nanoparticles without the addition of both Ce6 and $\mathrm{Ru}$ (III). To adjust the drug loading capacity, the concentration of the initial Ce6 was varied from 0 to $120 \mathrm{mg}$. The final products were centrifuged at $16099 \times \mathrm{g}$ for $30 \mathrm{~min}$, washed thrice with water and dispersed for further characterizations.

Decomposition of $\mathbf{H}_{\mathbf{2}} \mathbf{O}_{\mathbf{2}}$. The catalytic effect of MC-r and OxgeMCC-r toward hydrogen peroxide was tested by mixing MC-r ( $2 \mathrm{mM})$ or OxgeMCC-r $(2 \mathrm{mM})$ with $\mathrm{H}_{2} \mathrm{O}_{2}(1 \mathrm{mM})$ in $\mathrm{PBS}$ at room temperature. At predetermined time points, the solution $(50 \mu \mathrm{L})$ was collected and added to $\mathrm{Ti}\left(\mathrm{SO}_{4}\right)_{2}$ solution $(100 \mu \mathrm{L})^{21}$. The content of $\mathrm{H}_{2} \mathrm{O}_{2}$ was calculated through measuring the UV-vis absorbance at 405 $\mathrm{nm}$. For verifying the catalytic durability of OxgeMCC-r SAE toward $\mathrm{H}_{2} \mathrm{O}_{2}, \mathrm{H}_{2} \mathrm{O}_{2}$ solution was added repeatedly to the OxgeMCC-r SAE solution followed by measuring the catalytic efficiency under $\mathrm{pH}$ value of 6.5. An optical oxygen sensor (NeoFox, Ocean Optics, Inc.) was used to quantify the amount of evolving oxygen in the reaction system $\left(150 \mu \mathrm{M}\right.$ of $\mathrm{H}_{2} \mathrm{O}_{2}$ and $250 \mu \mathrm{M}$ of OxgeMCC-r). For comparison, $\mathrm{MnO}_{2}$ nanoparticles were also synthesized. Typically, bovine serum albumin $(100 \mathrm{mg})$ was added into $\mathrm{MnCl}_{2}$ solution $(10 \mathrm{~mL}, 10 \mathrm{mM})$. Thereafter, sodium hydroxide solution $(1.0 \mathrm{M})$ was added dropwise into the mixed solution to adjust the $\mathrm{pH}$ value to 11 . After vigorous stirring for $4 \mathrm{~h}, \mathrm{MnO}_{2}$ nanoparticles were obtained. The catalytic durability of $\mathrm{MnO}_{2}$ nanoparticles toward $\mathrm{H}_{2} \mathrm{O}_{2}$ was also studied under $\mathrm{pH}$ value of 6.5 .

Singlet oxygen detection. A singlet oxygen indicator 1,3-diphenylisobenzonfuran (DPBF, Sigma-Aldrich) was used to study ${ }^{1} \mathrm{O}_{2}$ generation by measuring the quenching UV-vis absorption at $421 \mathrm{~nm}$. The test solution was prepared by adding DPBF $(30 \mu \mathrm{L})$ in DMSO $(10 \mathrm{mM})$ to OxgeMCC-r solution $\left(3 \mathrm{~mL}, 10 \mu \mathrm{gL}^{-1}\right)$. Before the irradiation $\left(671 \mathrm{~nm}, 100 \mathrm{~mW} \mathrm{~cm}^{-2}, 30 \mathrm{~s}\right.$, light dose is $0.1 \mathrm{~J} \mathrm{~cm}^{-2} \mathrm{~s}^{-1}$ ), the mixed solution was saturated with argon atmosphere under dark environment to achieve hypoxic condition. Upon the irradiation, the absorption intensity of DPBF was recorded every 2 min. As controls, DPBF absorption was recorded in PBS, and MCC solution plus $\mathrm{H}_{2} \mathrm{O}_{2}$ with or without $671 \mathrm{~nm}$ irradiation.

In vitro PDT. Three cell lines including human embryonic kidney normal cells (HEK 293), human cervical cancer cells (HeLa), and murine breast cancer cells (4T1) were originally obtained from American Type Culture Collection (ATCC). Three cell lines were seeded in 96-well plates with an initial seeding density of $1 \times$ $10^{4}$ cells per well and incubated in $5 \% \mathrm{CO}_{2}$ at $37^{\circ} \mathrm{C}$ for $24 \mathrm{~h}$. For the biocompatibility study, the cells were incubated with MC-r, MCC, and OxgeMCC-r SAEs at different concentrations in the dark condition for $24 \mathrm{~h}$. For in vitro therapy, 4T1 cancer cells were incubated with free Ce6, MCC, and OxgeMCC-r at a range of concentrations at $37^{\circ} \mathrm{C}$ under normoxic or hypoxic condition for $24 \mathrm{~h}$. The hypoxic condition was achieved by culturing cells in an incubator supplied with hypoxic atmosphere $\left(\mathrm{N}_{2} / \mathrm{CO}_{2} / \mathrm{O}_{2}: 94 / 5 / 1\right.$ in volume ratio). After $4 \mathrm{~h}$ incubation, the cells were irradiated with a $671 \mathrm{~nm}$ laser $\left(100 \mathrm{~mW} \mathrm{~cm}^{-2}, 30 \mathrm{~s}\right)$. The relative cell viability was measured using the standard MTT assay. Typically, the sample-containing medium was replaced with MTT solution $\left(5 \mu \mathrm{g} \mathrm{mL} \mathrm{L}^{-1}, 10 \mu \mathrm{L}\right)$ and incubated for $4 \mathrm{~h}$. Finally, DMSO $(100 \mu \mathrm{L})$ was added into each well to dissolve the purple formazan crystals before measuring the UV-vis absorbance. Another mitochondrial activity-independent CellTiter ${ }^{\mathrm{TM}}$ Fluor cell viability assay (Promega Pte Ltd.) was also performed to study the in vitro therapeutic efficiency under both normoxic and hypoxic conditions. The dark toxicity was evaluated similarly but without light irradiation.

Live/dead cell staining assay. $4 \mathrm{~T} 1$ cancer cells were incubated in 6 -well plates under hypoxic condition at $37^{\circ} \mathrm{C}$ for $24 \mathrm{~h}$. After which, previous medium was replaced by fresh medium that contained free Ce6, MCC, and OxgeMCC-r (Ce6 concentration is $8 \mathrm{ppm}$ ) for $4 \mathrm{~h}$ co-culture. Thereafter, the cells were treated with a $671 \mathrm{~nm}$ laser irradiation $\left(100 \mathrm{~mW} \mathrm{~cm}^{-2}, 30 \mathrm{~s}\right)$. After co-culture for another $4 \mathrm{~h}$, cells were stained with calcein AM $(5 \mu \mathrm{L})$ and PI $(10 \mu \mathrm{L})$ according to the product description for $0.5 \mathrm{~h}$ and then observed using a fluorescence inverted microscope (Olympus UHGLGPS, China).

Apoptosis and necrosis assay. 4T1 cancer cells were seeded in 6-well plates under hypoxic condition at $37^{\circ} \mathrm{C}$ for $24 \mathrm{~h}$. Subsequently, previous medium was replaced by fresh one with free Ce6, MCC, and OxgeMCC-r ([Ce6] is 8 ppm). After $4 \mathrm{~h}$, the cells were irradiated with laser irradiation $\left(671 \mathrm{~nm}, 100 \mathrm{~mW} \mathrm{~cm}^{-2}, 30 \mathrm{~s}\right)$. After co-culture for another $4 \mathrm{~h}$, all treated cells were harvested and quantified by apoptosis with an annexin V-FITC/PI apoptosis detection kit using flow cytometer (Guava EasyCyte 6-2L).

Intracellular ROS detection. 4T1 cells were seeded with a density of $3.6 \times 10^{4}$ per well in a confocal dish under hypoxic condition. After the incubation at $37^{\circ} \mathrm{C}$ for $24 \mathrm{~h}$, previous medium was replaced by fresh one containing PBS, MCC, or OxgeMCC-r ([Ce6] is $8 \mathrm{ppm}$ ) in the dark, followed by the addition of cellpermeant 2',7'-dichlorodihydrofluorescein diacetate $\left(\mathrm{H}_{2}\right.$-DCFDA, $\left.25 \mu \mathrm{M}\right)$. After 4 $\mathrm{h}$, the cells were treated with laser irradiation $\left(671 \mathrm{~nm}, 100 \mathrm{~mW} \mathrm{~cm}^{-2}, 30 \mathrm{~s}\right)$. After culturing for another $4 \mathrm{~h}$, all treated groups were then observed using laser scanning confocal microscope (ZEISS, LSM710).

Intracellular hypoxia immunofluorescence. $4 \mathrm{~T} 1$ cells were seeded in confocal dish. Following different treatments, cells were fixed with $1 \%$ paraformaldehyde at $37^{\circ} \mathrm{C}$ for $10 \mathrm{~min}$ and permeabilized with PBS containing $0.2 \%$ Triton X-100 at $37^{\circ}$ $\mathrm{C}$ for $1 \mathrm{~min}$. Subsequently, the blocking step was carried out with PBST (PBS with $0.05 \%$ Tween-20) buffer containing $1 \%$ bovine serum albumin at room temperature for $45 \mathrm{~min}$. Finally, the cells were incubated with anti-HIF-1a (Cat. no. 36169 T, Cell Signaling Technology) and anti- $\alpha$-Tubulin (Cat. no. T9026, SigmaAldrich) primary antibodies in a humidified chamber at $4{ }^{\circ} \mathrm{C}$ overnight, followed by corresponding fluorescence labeled secondary antibodies at room temperature for $1 \mathrm{~h}$. Images were acquired with a DeltaVision softWoRx software (Applied Precision) and processed by deconvolution and $\mathrm{z}$-stack projection.

Western blot. 4T1 cells were first treated with MC-r before incubation under normoxic or hypoxic condition. After which, cells were lysed and collected, followed by mixing with sample buffer and heated at $95{ }^{\circ} \mathrm{C}$ for $5 \mathrm{~min}$. After the purification with $10 \%$ sodium dodecyl sulfate-polyacrylamide gel electrophoresis, the collected protein was transferred to a polyvinylidene difluoride membrane. The 
sample was stained with primary antibodies anti-HIF-1 $\alpha$ (Cat no. 36169T, Cell Signaling Technology) for evaluating the degree of hypoxia and anti- $\alpha$-Tubulin (Cat. no. T9026, Sigma-Aldrich) as the loading control, and then with horseradish peroxidase-labeled secondary antibodies. The HIF-1 $\alpha$ level was monitored by enhanced chemiluminescence using Gel Doc system (Bio-Rad).

Intracellular $\mathbf{O}_{\mathbf{2}}$ generation. $4 \mathrm{~T} 1$ cells were first seeded on cover slides and incubated with PBS or MC-r under normal or hypoxic condition for $12 \mathrm{~h}$. Following which, cells were treated with an oxygen indicator $\left[\mathrm{Ru}(\mathrm{dpp})_{3}\right] \mathrm{Cl}_{2}(10 \mu \mathrm{g} /$ $\mathrm{mL}$, ThermoFisher) and further incubated for $12 \mathrm{~h}$. The samples were removed and cells were washed thrice with PBS to remove any residual MC-r nanoparticles and free $\left[\mathrm{Ru}(\mathrm{dpp})_{3}\right] \mathrm{Cl}_{2}$ molecules. Fluorescence images of $\left[\mathrm{Ru}(\mathrm{dpp})_{3}\right]^{2+}\left(\lambda_{\mathrm{ex}}=450 \mathrm{~nm}\right.$, $\lambda_{\mathrm{em}}=610 \mathrm{~nm}$ ) relating to the intracellular $\mathrm{O}_{2}$ level was obtained using a confocal laser scanning microscope.

MR imaging. OxgeMCC-r SAE solutions with different Mn concentrations were measured with a clinical MR imaging scanner (GE HDxt, 3.0-T) from the First Affiliated Hospital of Anhui Medical University. For in vitro MR imaging, 4T1 cells were pretreated with $18.4 \mathrm{ppm}$ OxgeMCC-r SAE. After incubation for 2 and $6 \mathrm{~h}$, $5 \times 10^{6}$ cells were washed with PBS three time and collected for MR imaging test. For in vivo MR imaging, 4T1 tumor-bearing mice $(n=3)$ weighing $20 \mathrm{~g}$ on average (SLAC Laboratory animal Co., Ltd., Shanghai) were employed for the study. Animal experiments were conducted under the animal guidelines authorized by the Animal Care Committee (University of Science and Technology of China) and complied with all relevant ethical regulations. $\mathrm{T}_{1}$-weighted magnetic resonance images were acquired pre-injection as well as $2 \mathrm{~h}, 6 \mathrm{~h}, 24 \mathrm{~h}$, and $48 \mathrm{~h}$ post-injection of OxgeMCC-r saline solution (13.2 $\mathrm{mg} \mathrm{kg}^{-1}$ ). Fast spin echo multi-slice (f-SEMS) sequence was used for the acquisition of the $\mathrm{T}_{1}$-weighted magnetic resonance images.

In vivo biocompatibility analysis. For the long-term biocompatibility study, mice were first intravenously injected with OxgeMCC-r SAE. Two-week later, the mice were euthanized and their main organs (heart, kidney, liver, lung, spleen, and intestine) were collected and fixed with $4 \%$ paraformaldehyde. After embedded in paraffin, tissue samples were cryo-sliced $(4 \mu \mathrm{m})$ before further histological analysis by standard hematoxylin and eosin (H\&E) staining procedure.

In vivo PDT. 4T1 tumor-bearing mice were randomly allocated into four groups $(n=5)$ : control, free Ce6, MCC, and OxgeMCC-r SAE when the average tumor volume reached $100 \mathrm{~mm}^{3}$. Ce6, MCC and OxgeMCC-r in saline solutions (injection dose $=100 \mu \mathrm{L}$, at a Ce6 concentration of $4 \mathrm{mg} \mathrm{kg}^{-1}$ ) were injected via the tail vein. For comparison, saline $(100 \mu \mathrm{L})$ was injected into mice as the control group. At $6 \mathrm{~h}$ post injection, the tumors from groups $2-4$ were irradiated with a $671 \mathrm{~nm}$ laser $\left(100 \mathrm{~mW} \mathrm{~cm}^{-2}, 5 \mathrm{~min}\right)$. Tumor sizes were measured every day since the start of treatment, and the tumor volume was calculated according to the equation: Volume $=($ Tumor length $) \times(\text { Tumor width })^{2} / 2\left(\mathrm{~mm}^{3}\right)$. Relative tumor volume was normalized to its initial size before the treatment. At the end of the treatment, all mice were sacrificed, and tumors were collected and weighed.

Immunofluorescence staining. The variation of hypoxia degree within tumor microenvironment after $24 \mathrm{~h}$ post injection of OxgeMCC-r SAE was investigated with immunofluorescence staining. At $90 \mathrm{~min}$ before tumors were surgically excised, pimonidazole hydrochloride (Hypoxyprobe-1 plus kit, Hypoxyprobe, USA) was intraperitoneally injected into mice at a dose of $30 \mathrm{mg} \mathrm{kg}^{-1}$. The collected tumor slices were firstly stained with mouse anti-pimonidazole monoclonal antibody (dilution 1:200, Hypoxyprobe) and rat-anti mouse CD31 primary antibody (dilution 1:200, Biolegend) to mark tumor hypoxia regions and blood vessels, respectively. Thereafter, the slices were stained with Alex 488-conjugated goat antimouse secondary antibody (Jackson Inc.) and rhodamine-conjugated donkey antirat secondary antibody (Jackson Inc.), respectively. The nuclei were stained with 4',6-diamidino-2-phenylindole (DAPI, Invitrogen). The final images of stained slices were obtained using a confocal laser scanning microscope (Zeiss LSM 710).

Pathological investigation. After in vivo therapy, tumor tissues of control and OxgeMCC-r groups were resected, fixed with $4 \%$ formaldehyde solution and embedded in paraffin blocks. The tissue blocks were sectioned and stained with hematoxylin and eosin (H\&E). For Ki-67 staining, tissue sections were stained with anti-Ki-67 polyclonal antibody (ab15580, Abcam, USA) at $4{ }^{\circ} \mathrm{C}$ overnight before imaging. Terminal deoxynucleotidyl transferase (TdT)-mediated deoxyuridine triphosphate (dUTP) nick end labeling (TUNEL) staining was conducted using in situ cell death detection kit (POD, Roche, America). All processes were carried out following the standard protocol.

Statistical analysis. All data were expressed as mean \pm standard error of the mean. The statistical difference between different groups of data was evaluated by oneway ANOVA, and $p<0.05$ was considered to be statistically significant. Asterisk $\left.{ }^{*}\right)$ denotes statistical significance between bars $\left({ }^{*} p<0.05,{ }^{* *} p<0.01,{ }^{* *} p<0.001\right)$ conducted using GraphPad Prism 6.0.
Reporting summary. Further information on research design is available in the Nature Research Reporting Summary linked to this article.

\section{Data availability}

The authors declare that the data supporting the findings of this study are available within the article and its Supplementary Information. Extra data are available from the corresponding author upon reasonable request.

Received: 11 June 2019; Accepted: 19 December 2019; Published online: 17 January 2020

\section{References}

1. Felsher, D. W. Cancer revoked: oncogenes as therapeutic targets. Nat. Rev. Cancer 3, 375-380 (2003).

2. Lucky, S. S., Soo, K. C. \& Zhang, Y. Nanoparticles in photodynamic therapy Chem. Rev. 115, 1990-2042 (2015).

3. Hopper, C. Photodynamic therapy: a clinical reality in the treatment of cancer. Lancet Oncol. 1, 212-219 (2000).

4. Lovell, J. F., Liu, T. W. B., Chen, J. \& Zheng, G. Activatable photosensitizers for imaging and therapy. Chem. Rev. 110, 2839-2857 (2010).

5. Tian, B., Wang, C., Zhang, S., Feng, L. \& Liu, Z. Photothermally enhanced photodynamic therapy delivered by nano-graphene oxide. ACS Nano $\mathbf{5}$, 7000-7009 (2011).

6. Abbas, M., Zou, Q., Li, S. \& Yan, X. Self-assembled peptide- and protein-based nanomaterials for antitumor photodynamic and photothermal therapy. $A d v$ Mater. 29, 1605021 (2017).

7. Xu, S. et al. Oxygen and Pt(II) self-generating conjugate for synergistic photochemo therapy of hypoxic tumor. Nat. Commun. 9, 2053 (2018)

8. Zhang, C. et al. Marriage of scintillator and semiconductor for synchronous radiotherapy and deep photodynamic therapy with diminished oxygen dependence. Angew. Chem. Int. Ed. 54, 1770-1774 (2015).

9. Xu, R. et al. Nanoscale metal-organic frameworks for ratiometric oxygen sensing in live cells. J. Am. Chem. Soc. 138, 2158-2161 (2016).

10. Vander Heiden, M. G., Cantley, L. C. \& Thompson, C. B. Understanding the Warburg effect: the metabolic requirements of cell proliferation. Science $\mathbf{3 2 4}$ 1029-1033 (2009).

11. Bristow, R. G. \& Hill, R. P. Hypoxia, DNA repair and genetic instability. Nat Rev. Cancer 8, 180 (2008)

12. Yu, Z., Zhou, P., Pan, W., Li, N. \& Tang, B. A biomimetic nanoreactor for synergistic chemiexcited photodynamic therapy and starvation therapy against tumor metastasis. Nat. Commun. 9, 5044 (2018).

13. Kuang, Y., Balakrishnan, K., Gandhi, V. \& Peng, X. Hydrogen peroxide inducible DNA cross-linking agents: targeted anticancer prodrugs. J. Am. Chem. Soc. 133, 19278-19281 (2011).

14. Szatrowski, T. P. \& Nathan, C. F. Production of large amounts of hydrogen peroxide by human tumor cells. Cancer Res. 51, 794-798 (1991).

15. Lambeth, J. D. NOX enzymes and the biology of reactive oxygen. Nat. Rev. Immunol. 4, 181-189 (2004).

16. Fan, W. et al. Intelligent $\mathrm{MnO}_{2}$ nanosheets anchored with upconversion nanoprobes for concurrent $\mathrm{pH}-/ \mathrm{H}_{2} \mathrm{O}_{2}$-responsive UCL imaging and oxygenelevated synergetic therapy. Adv. Mater. 27, 4155-4161 (2015).

17. Huang, C. C. et al. An implantable depot that can generate oxygen in situ for overcoming hypoxia-induced resistance to anticancer drugs in chemotherapy. J. Am. Chem. Soc. 138, 5222-5225 (2016)

18. Jia, Q. et al. A magnetofluorescent carbon dot assembly as an acidic $\mathrm{H}_{2} \mathrm{O}_{2}$ driven oxygenerator to regulate tumor hypoxia for simultaneous bimodal imaging and enhanced photodynamic therapy. Adv. Mater. 30, 1706090 (2018).

19. Wang, H. et al. Photosensitizer-crosslinked in-situ polymerization on catalase for tumor hypoxia modulation \& enhanced photodynamic therapy. Biomaterials 181, 310-317 (2018).

20. Kim, J. et al. Continuous $\mathrm{O}_{2}$-evolving $\mathrm{MnFe}_{2} \mathrm{O}_{4}$ nanoparticle-anchored mesoporous silica nanoparticles for efficient photodynamic therapy in hypoxic cancer. J. Am. Chem. Soc. 139, 10992-10995 (2017).

21. Wang, D. et al. Photo-enhanced singlet oxygen generation of Prussian bluebased nanocatalyst for augmented photodynamic therapy. iScience 9, 14-26 (2018).

22. Yang, G. et al. Hollow $\mathrm{MnO}_{2}$ as a tumor-microenvironment-responsive biodegradable nano-platform for combination therapy favoring antitumor immune responses. Nat. Commun. 8, 902 (2017).

23. Wei, S. et al. Direct observation of noble metal nanoparticles transforming to thermally stable single atoms. Nat. Nanotechnol. 13, 856-861 (2018).

24. Wang, A., Li, J. \& Zhang, T. Heterogeneous single-atom catalysis. Nat. Rev. Chem. 2, 65-81 (2018). 
25. Lin, L. et al. Low-temperature hydrogen production from water and methanol using Pt/alpha-MoC catalysts. Nature 544, 80-83 (2017).

26. Liu, G. et al. $\mathrm{MoS}_{2}$ monolayer catalyst doped with isolated Co atoms for the hydrodeoxygenation reaction. Nat. Chem. 9, 810-816 (2017).

27. Geng, Z. et al. Achieving a record-high yield rate of $120.9 \mu \mathrm{g}_{\mathrm{NH} 3} \mathrm{mg}_{\text {cat. }}{ }^{-1} \mathrm{~h}^{-1}$ for $\mathrm{N}_{2}$ electrochemical reduction over Ru single-atom catalysts. Adv. Mater. 30, 1803498 (2018).

28. Gong, N. et al. Carbon-dot-supported atomically dispersed gold as a mitochondrial oxidative stress amplifier for cancer treatment. Nat. Nanotechnol. 14, 379-387 (2019).

29. $\mathrm{Xu}, \mathrm{B}$. et al. A single-atom nanozyme for wound disinfection applications. Angew. Chem. Int. Ed. 58, 4911-4916 (2019).

30. Wang, D. et al. Controllable synthesis of dual-MOFs nanostructures for $\mathrm{pH}$ responsive artemisinin delivery, magnetic resonance and optical dual-model imaging-guided chemo/photothermal combinational cancer therapy. Biomaterials 100, 27-40 (2016)

31. Cheng, G. et al. Self-assembly of extracellular vesicle-like metal-organic framework nanoparticles for protection and intracellular delivery of biofunctional proteins. J. Am. Chem. Soc. 140, 7282-7291 (2018).

32. Zheng, H. et al. One-pot synthesis of metal-organic frameworks with encapsulated target molecules and their applications for controlled drug delivery. J. Am. Chem. Soc. 138, 962-968 (2016).

33. Rogge, S. M. J. et al. Metal-organic and covalent organic frameworks as singlesite catalysts. Chem. Soc. Rev. 46, 3134-3184 (2017).

34. Wu, M. X. \& Yang, Y. W. Metal-organic framework (MOF)-based drug/cargo delivery and cancer therapy. Adv. Mater. 29, 1606134 (2017).

35. Otake, K. I. et al. Single-atom-based vanadium oxide catalysts supported on metal-organic frameworks: selective alcohol oxidation and structure-activity relationship. J. Am. Chem. Soc. 140, 8652-8656 (2018).

36. Wang, D. et al. Core-shell metal-organic frameworks as $\mathrm{Fe}^{2+}$ suppliers for $\mathrm{Fe}^{2+}$-mediated cancer therapy under multimodality imaging. Chem. Mater. 29, 3477-3489 (2017).

37. Wang, Y. et al. Universal strategy for homogeneously doping noble metals into cyano-bridged coordination polymers. ACS Appl. Mater. Interfaces 7, 2088-2096 (2015).

38. Jiang, P. et al. Tuning the activity of carbon for electrocatalytic hydrogen evolution via an iridium-cobalt alloy core encapsulated in nitrogen-doped carbon cages. Adv. Mater. 30, 1705324 (2018).

39. Su, J. et al. Ruthenium-cobalt nanoalloys encapsulated in nitrogen-doped graphene as active electrocatalysts for producing hydrogen in alkaline media. Nat. Commun. 8, 14969 (2017).

40. Cao, G. J., Jiang, X., Zhang, H., Croley, T. R. \& Yin, J. J. Mimicking horseradish peroxidase and oxidase using ruthenium nanomaterials. RSC Adv. 7, 52210-52217 (2017).

41. Zhang, Y. et al. Nanozyme decorated metal-organic frameworks for enhanced photodynamic therapy. ACS Nano 12, 651-661 (2018).

42. Jv, Y., Li, B. \& Cao, R. Positively-charged gold nanoparticles as peroxidase mimic and their application in hydrogen peroxide and glucose detection. Chem. Commun. 46, 8017-8019 (2010).

43. Wang, D. et al. In situ one-pot synthesis of MOF-polydopamine hybrid nanogels with enhanced photothermal effect for targeted cancer therapy. Adv. Sci. 5, 1800287 (2018).

44. Wang, D. et al. Novel $\mathrm{Mn}_{3}\left[\mathrm{Co}(\mathrm{CN})_{6}\right]_{2} @ \mathrm{SiO}_{2} @ \mathrm{Ag}$ core-shell nanocube: enhanced two-photon fluorescence and magnetic resonance dual-modal imaging-guided photothermal and chemo-therapy. Small 11, 5956-5967 (2015).

45. Tao, H. et al. Nitrogen fixation by Ru single-atom electrocatalytic reduction. Chem 5, 204-214 (2019).

46. Yabuta, H. et al. X-ray absorption near edge structure spectroscopic study of Hayabusa category 3 carbonaceous particles. Earth, Planets Space 66, 156 (2014).

47. Wang, X. et al. Uncoordinated amine groups of metal-organic frameworks to anchor single $\mathrm{Ru}$ sites as chemoselective catalysts toward the hydrogenation of quinoline. J. Am. Chem. Soc. 139, 9419-9422 (2017).

48. Nowicki, A., Le Boulaire, V. \& Roucoux, A. Nanoheterogeneous catalytic hydrogenation of arenes: evaluation of the surfactant-stabilized aqueous ruthenium(0) colloidal suspension. Adv. Synth. Catal. 349, 2326-2330 (2007).

49. Bonet, F. et al. Synthesis of monodisperse $\mathrm{Au}, \mathrm{Pt}, \mathrm{Pd}, \mathrm{Ru}$ and $\mathrm{Ir}$ nanoparticles in ethylene glycol. Nanostruct. Mater. 11, 1277-1284 (1999).

50. Zhang, C. et al. Magnesium silicide nanoparticles as a deoxygenation agent for cancer starvation therapy. Nat. Nanotechnol. 12, 378-386 (2017).

51. Lopes, C. M. A. \& Felisberti, M. I. Mechanical behaviour and biocompatibility of poly(1-vinyl-2-pyrrolidinone)-gelatin IPN hydrogels. Biomaterials 24, 1279-1284 (2003).
52. Chen, Q. et al. Intelligent albumin- $\mathrm{MnO}_{2}$ nanoparticles as $\mathrm{pH}-/ \mathrm{H}_{2} \mathrm{O}_{2}$ responsive dissociable nanocarriers to modulate tumor hypoxia for effective combination therapy. Adv. Mater. 28, 7129-7136 (2016).

53. Salceda, S. \& Caro, J. Hypoxia-inducible factor $1 \alpha$ (HIF-1a) protein is rapidly degraded by the ubiquitin-proteasome system under normoxic conditions: its stabilization by hypoxia depends on redox-induced changes. J. Biol. Chem. 272, 22642-22647 (1997).

54. Huo, M., Wang, L., Chen, Y. \& Shi, J. Tumor-selective catalytic nanomedicine by nanocatalyst delivery. Nat. Commun. 8, 357 (2017).

55. Hifumi, H., Yamaoka, S., Tanimoto, A., Citterio, D. \& Suzuki, K. Gadoliniumbased hybrid nanoparticles as a positive MR contrast agent. J. Am. Chem. Soc. 128, 15090-15091 (2006)

56. Fang, J., Nakamura, H. \& Maeda, H. The EPR effect: unique features of tumor blood vessels for drug delivery, factors involved, and limitations and augmentation of the effect. Adv. Drug Deliv. Rev. 63, 136-151 (2011).

57. Zhu, P., Chen, Y. \& Shi, J. Nanoenzyme-augmented cancer sonodynamic therapy by catalytic tumor oxygenation. ACS Nano 12, 3780-3795 (2018).

58. Wang, D. et al. Magnetically guided delivery of DHA and Fe ions for enhanced cancer therapy based on $\mathrm{pH}$-responsive degradation of DHA-loaded $\mathrm{Fe}_{3} \mathrm{O}_{4} @ \mathrm{C} @ \mathrm{MIL}-100(\mathrm{Fe})$ nanoparticles. Biomaterials 107, 88-101 (2016).

\section{Acknowledgements}

The work was supported by the Singapore National Research Foundation Investigatorship (NRF-NRFI2018-03), the MOST Grant (2016YFA0101202) and the National Natural Science Foundation of China (31471268). The authors thank Dr. Jianwei Su and Dr. Peng Jiang for kind help and valuable suggestions. The authors also acknowledge the Soft X-Ray Magnetic Circular Dichroism End Station (XMCD) of Hefei National Synchrotron Radiation Laboratory (NSRL) for testing the near-edge X-ray absorption fine structure (NEXAFS) of C K-edge and N K-edge.

\section{Author contributions}

Y.Z., Z.G. and D.W. conceived the idea and designed the project. D.W., H.H.W., G.Y., C.Q., H.B.W. and Z.G. performed the experiments and analyzed the results. L.G. and H.C. assisted with the figure production and experiment design. Y.Z. and D.W. wrote and revised the original draft of the manuscript. Y.Z., D.W., S.Z.F.P and W.Q.L. edited the manuscript. Y.Z. supervised the whole project. All authors discussed the results and commented on the manuscript.

\section{Competing interests}

The authors declare no competing interests.

\section{Additional information}

Supplementary information is available for this paper at https://doi.org/10.1038/s41467 019-14199-7.

Correspondence and requests for materials should be addressed to Z.G. or Y.Z.

Peer review information Nature Communications thanks Mateusz Kwitniewski, Zhuang Liu and the other, anonymous, reviewer(s) for their contribution to the peer review of this work. Peer reviewer reports are available.

Reprints and permission information is available at http://www.nature.com/reprints

Publisher's note Springer Nature remains neutral with regard to jurisdictional claims in published maps and institutional affiliations.

Open Access This article is licensed under a Creative Commons Attribution 4.0 International License, which permits use, sharing, adaptation, distribution and reproduction in any medium or format, as long as you give appropriate credit to the original author(s) and the source, provide a link to the Creative Commons license, and indicate if changes were made. The images or other third party material in this article are included in the article's Creative Commons license, unless indicated otherwise in a credit line to the material. If material is not included in the article's Creative Commons license and your intended use is not permitted by statutory regulation or exceeds the permitted use, you will need to obtain permission directly from the copyright holder. To view a copy of this license, visit http://creativecommons.org/ licenses/by/4.0/

(C) The Author(s) 2020 